\title{
Differential Density Statistics of Galaxy Distribution and the Luminosity Function
}

\author{
V. V. L. Albani, ${ }^{1}$ A. S. Iribarrem, ${ }^{2}$ M. B. Ribeiro ${ }^{2}$ \\ University of Brazil-UFRJ, Rio de Janeiro \\ and W. R. Stoeger ${ }^{3}$ \\ Vatican Observatory, University of Arizona, Tucson
}

\begin{abstract}
This paper uses data obtained from the galaxy luminosity function (LF) to calculate two types of radial number densities statistics of the galaxy distribution as discussed in Ribeiro (2005), namely the differential density $\gamma$ and the integral differential density $\gamma^{*}$. By applying the theory advanced by Ribeiro and Stoeger (2003), which connects the relativistic cosmology number counts with the astronomically derived LF, the differential number counts $d N / d z$ are extracted from the LF and used to calculate both $\gamma$ and $\gamma^{*}$ with various cosmological distance definitions, namely the area distance, luminosity distance, galaxy area distance and redshift distance. LF data are taken from the CNOC2 galaxy redshift survey and $\gamma$ and $\gamma^{*}$ are calculated for two cosmological models: Einstein-de Sitter and an $\Omega_{m_{0}}=0.3, \Omega_{\Lambda_{0}}=0.7$ standard cosmology. The results confirm the strong dependency of both statistics on the distance definition, as predicted in Ribeiro (2005), as well as showing that plots of $\gamma$ and $\gamma^{*}$ against the luminosity and redshift distances indicate that the $\mathrm{CNOC} 2$ galaxy distribution follows a power law pattern for redshifts higher than 0.1. These findings bring support to Ribeiro's (2005) theoretical proposition that using different cosmological distance measures in statistical analyses of galaxy surveys can lead to significant ambiguity in drawing conclusions about the behavior of the observed large scale distribution of galaxies.
\end{abstract}

Subject headings: galaxies: luminosity function, mass function — relativity

\footnotetext{
${ }^{1}$ Mathematics Institute-UFRJ

${ }^{2}$ Physics Institute, University of Brazil - UFRJ, CxP 68532, CEP 21945-970, Rio de Janeiro, Brazil

${ }^{3}$ Vatican Observatory Group, Steward Observatory, University of Arizona, Tucson, AZ 85721, USA
} 


\section{Introduction}

The galaxy luminosity function (LF) is an important analytical tool for testing cosmological models. It conveys observational information on how the local density of galaxies varies for different types of galaxies and with differing cosmic environments, as well as how the local galaxy density changes with cosmic epoch. The LF has been extensively and systematically calculated for a great variety of galaxy redshift surveys, in terms of the number of galaxies, their morphological types, observational bandwidths and redshift depths. In addition, since in calculating the LF one often includes a model of source evolution, the presently available LF parameters obtained from different galaxy samples provide an important collection of data for testing cosmological models. To do so, however, we need a detailed theoretical framework connecting the astronomical LF data calculation and practice to cosmological modeling. This is essential for extracting information that can be used for further constraining cosmological models.

In a recent paper, Ribeiro and Stoeger (2003; from now on RS03) have presented a detailed relativistic treatment directly linking the cosmology source counting theory with LF data. RS03 started with a general relativistic treatment of number source counts in a general spacetime and then derived expressions for relativistic density per source and total relativistic energy densities of the universe in terms of the luminosity and selection functions. These theoretical/observational relationships were applied to test the LF parameters determined from the CNOC2 galaxy redshift survey (Lin et al. 1999) for consistency with the assumed Einstein-de Sitter (EdS) cosmological model. Other possible applications and extensions of this general framework were also outlined.

This present work explores one of these extensions of RS03. First, we shall show how to extract the differential number counts $d N / d z$ from LF data. Inasmuch as calculating the LF parameters from a galaxy sample requires the assumption of a cosmological model, since a volume definition, usually comoving, must be employed, one can factor the cosmology out of the LF parameters and re-obtain the "raw" number count data. The methodology for determining LF's requires fitting the original data to some analytical form of the LF, usually of the Schechter type, where a model of source evolution is also included. Therefore, by "raw' number count data" we mean that the reverse process eliminates the cosmology, but does not remove the fitting itself, that is, the LF analytical assumption and source evolution corrections.

Once these "raw" $d N / d z$ are in our possession, we carry out a statistical analysis with the differential density parameters discussed in Ribeiro (2005), which are basically different forms of number densities. As such this analysis is based on the differential number counts. Thus, it can be done in any cosmological model (since the $d N / d z$ data does not have any 
kind of cosmology built into it). Here we test the theoretical proposition advanced in Ribeiro (2005) that using different cosmological distance measures in statistical analyses of galaxy surveys can lead to significant ambiguity in drawing conclusions about the behavior of the observed large scale distribution of galaxies.

Besides the sensitivity of these large scale analyses to the cosmological distances used, comparison of number counts at different redshifts is made more difficult by the fact that, as we look down our past light cone, we are counting galaxies at different redshifts, and therefore at different times. Our line of sight cuts across many different space-like, possibly nearly spatially homogeneous, surfaces. This means, that, even aside from the distance measure employed, the number counts down our light cone will not correspond in any simple way with the integrated galaxy number density over a volume on any space-like three-surface.

Thus, even though there is "almost homogeneity" 4 of the distribution of galaxies, and therefore of mass-energy, on spatial 3-surfaces (Peebles 1993 and references therein, Hogg et al. 2005), we should not expect to find "observational homogeneity" as we count galaxies down our past light cone. Yet, employing some distance measures (e.g. the co-moving distance and the galaxy area distance) leads to the appearance of such observational homogeneity. For other measures of distance, we obtain, as Ribeiro (2005) has shown theoretically and as we here shall show from the CNOC2 data, a power-law galaxy distribution down the light cone, indicating a possible self-similar, hierarchical clustering or fractal-like pattern even though the actual spatial distribution is almost homogeneous. This is simply the resulting effect of collecting data from successively earlier epochs as we go to larger redshifts.

Besides showing how to extract cosmology-free $d N / d z$ information from LF's, a primary objective of the paper is to clarify these issues, emphasizing the illusory and ambiguous character of such observational homogeneity and of such purely observational galaxy distributions, in general.

The main conclusions we reach in this paper are as follows. The galaxy distribution given in the CNOC2 redshift survey supports Ribeiro's (2005) theoretical finding that the statistics of the observed galaxy distribution are strongly dependent on the choice of cosmological distance definitions. Both the differential density $\gamma$, which gives the rate of increase in galaxy number counts with distance down the past light cone, and the integral differential density $\gamma^{*}$, which is simply the integral of $\gamma$ over a specified volume, decay with higher

\footnotetext{
4 We use this term to describe succinctly one of the key characteristics of a perturbed FriedmannLemaitre-Robertson-Walker (FLRW) universe. Our universe is obviously not exactly spatially homogeneous - otherwise we would not be here! But there is strong evidence that it is "almost homogeneous," or "almost FLRW," on very large scales.
} 
luminosity and redshift distances, with no flattening of this decrease being found within the limits of the CNOC2 survey $(0 \leq z \leq 1)$. These decreasing values of the differential densities show a tendency to linearization in a $\log -\log$ plot for $z>0.1$, that is, a power law pattern, which suggests a self-similar behavior for the observed galaxy distribution in those ranges. Again, it is important to recognize that these parameters are measuring changes in galaxy number density down our past light cone, not in the space-like surfaces of the Friedmann-Lemaitre-Robertson-Walker (FLRW) models where there is evidence of "almost homogeneity". These results are valid for both EdS cosmology and the currently favored FLRW standard model with $\Omega_{m_{0}}=0.3$ and $\Omega_{\Lambda_{0}}=0.7$. However, by comparing the results of both models it seems that the linearization tendency mentioned above is more pronounced in the second model, suggesting that FLRW cosmologies with a less than critical matter density parameter might be a better fit as far as a self-similar distribution is concerned.

The plan of the paper is as follows. In $\S 2$ the main equations, derived in RS03, necessary for our purposes here are summarized and the method for extracting the differential number count $d N / d z$ from LF data is developed and applied to the CNOC2 galaxy redshift survey data. In $\S 3$ we calculate the differential density statistics in two cosmological models, namely EdS and the presently favored standard model with $\Omega_{m_{0}}=0.3, \Omega_{\Lambda_{0}}=0.7$. $\S 4$ summarizes our conclusions and the appendix briefly describes the error analysis performed with the CNOC2 data.

\section{Differential Number Counts from LF Data}

In this section we present a summary of the relevant results from RS03 and derive the general equation for the observed differential number count. Then we will specialize these equations to the EdS model in order to be able to obtain the "raw" $d N / d z$ from the LF parameters calculated with the CNOC2 galaxy redshift survey.

\subsection{General Relations}

We start with the general equation for the number of cosmological sources $N$ in a volume section at a certain point down the null cone whose affine parameter distance is given by $y$,

$$
d N=\left(d_{A}\right)^{2}(1+z) d \Omega_{0} n(y) d y .
$$

Here $d_{A}$ is the area distance (also known as angular diameter distance, observer area distance or corrected luminosity distance) of this section from the observer's viewpoint, $d \Omega_{0}$ is the solid angle of the volume section at the observer's position, $n$ is the number density of 
radiating sources per unit proper volume and $z$ is the redshift. This equation is simply a more convenient rewriting of Ellis' (1971) key result (see also Plebański and Krasiński 2006) for cosmological number counts in any cosmological model (see details in RS03, §2.1.1). The number density $n$ appearing in the equation above is related to the galaxy luminosity function $\phi$ as follows,

$$
n(y)=\Psi[z(y)]=\int_{0}^{\infty} \phi(l) d l
$$

where $l=L / L_{*}, L$ is the absolute luminosity of the source and $L_{*}$ is the luminosity scale parameter (RS03, eqs. 29, 35). In addition we have that

$$
\rho=\mathcal{M}_{g} \Psi
$$

where $\rho$ is the local density as given by the right-hand side of Einstein's field equations and $\mathcal{M}_{g}$ is the average galaxy rest mass (RS03, $\S 2.1 .2$, eq. 40). Therefore, equation (1) can be written as,

$$
\frac{d N}{d z}=\left(d_{A}\right)^{2}(1+z) d \Omega_{0}\left(\frac{\rho}{\mathcal{M}_{g}}\right) \frac{d y}{d z}
$$

This equation can be directly written in terms of observable quantities in a certain bandwidth $W$ as follows (RS03, $§ 4.4$, eqs. 46, 84),

$$
\left[\frac{d N}{d z}\right]_{0}=\sum_{W} a_{W}\left[\frac{d N}{d z}\right]^{W}
$$

Here the subscript zero means an observed quantity, and the superscript index $W$ means the observational bandwidth used when the data was collected. The factor $a_{W}$ is the fraction of galaxies in a certain wave band that are not counted in other bandwidths. This is necessary to avoid overcounting due to possible overlap of galaxy counts in different bandwidths. Therefore, we have that (RS03, eqs. 45, 46),

$$
a_{W}(z)= \begin{cases}1, & \text { for } W=1 \\ b_{W}(z)<1, & \text { for } W>1\end{cases}
$$

where, $b_{W}(z)$ is the fraction of galaxies in each waveband, that is, when $W>1$, which are not counted in wavebands $1,2, \ldots,(W-1)$. Thus, equation (4) can also be written

$$
\left[\frac{d N}{d z}\right]_{0}=\left(d_{A}\right)^{2}(1+z) d \Omega_{0} \frac{d y}{d z}\left[\frac{\rho}{\mathcal{M}_{g}}\right]_{0}=\left(d_{A}\right)^{2}(1+z) d \Omega_{0} \frac{d y}{d z} \sum_{W} a_{W}\left[\frac{\rho}{\mathcal{M}_{g}}\right]^{W}
$$

Similarly, equation (3) yields

$$
\rho_{0}=\left[\Psi \mathcal{M}_{g}\right]_{0}=\sum_{W} a_{W}\left(\psi^{W} \mathcal{M}_{g}\right)=\sum_{W} a_{W} \sum_{v} P_{v}^{W} \mathcal{M}_{v} \psi_{v}^{W} .
$$


Here $\psi^{W}$ is the selection function calculated from the luminosity function obtained in a certain wave band $W$,

$$
\psi^{W}[l(z)]=\int_{l(z)}^{\infty} \phi^{W}(l) d l,
$$

$P_{v}^{W}$ is the galactic morphology population fraction, giving the abundance of each galactic type relative to the total number of counted galaxies in each redshift range and observed in a certain wave band $W$. Finally, $\mathcal{M}_{v}$ is the average rest-mass for galaxies of a certain morphological class $v$ (see eqs. 13, 14, 87 of RS03).

From the discussion above it is clear that it is still possible to write the observed ratio between the local density and the average galaxy rest-mass as follows,

$$
\left[\frac{\rho}{\mathcal{M}_{g}}\right]^{W}=\frac{\sum_{v} P_{v}^{W} \mathcal{M}_{v} \psi_{v}^{W}}{\sum_{v} P_{v}^{W} \mathcal{M}_{v}}
$$

This equation allows us to write the observed differential number counts in terms of the chosen cosmological model and observational quantities extractable from galaxy catalogues. This result may be written as below,

$$
\left[\frac{d N}{d z}\right]_{0}=\left(d_{A}\right)^{2}(1+z) d \Omega_{0} \frac{d y}{d z} \sum_{W} a_{W}\left[\frac{\sum_{v} P_{v}^{W} \mathcal{M}_{v} \psi_{v}^{W}}{\sum_{v} P_{v}^{W} \mathcal{M}_{v}}\right] .
$$

Notice again that this equation is absolutely general, independent of cosmological model and independent of the survey, since its observational part under the summation signs is written in such a way as to be applicable, in principle, to any galaxy sample (RS03, §3.1).

\subsection{Evolution of Galaxy Masses}

A straightforward examination of equation (11) shows that it depends on the typical average rest-mass $\mathcal{M}_{v}$ of galaxies belonging to the morphological class $v$. The way $\mathcal{M}_{v}$ appears in this equation might indicate that this typical mass remains unchanged for different redshifts, a hypothesis which is certainly unrealistic as there is today evidence for galaxy mergers. Therefore, we must consider some dependency of the typical galaxy mass with the redshift in order to have a realistic assumption.

One way of doing this would be by replacing $\mathcal{M}_{v}$ in equation $(11)$ by $\left[f(z) \widetilde{\mathcal{M}}_{v}\right]$, where $\widetilde{\mathcal{M}}_{v}$ is the typical mass of $v$ type galaxy at $z=0$, that is, at rest with us, or here and now, and such that the function $f(z)$ obeys $f(0)=1$. So, if a galaxy doubles its mass from $z=1$ to $z=0$ we could take $f(z)=(1+z)^{-1}$. A milder growth would be achieved by assuming $f(z)=(1+z)^{-q}$, with $0<q<1$. So, for $q=0.5$ the galaxy would have $70 \%$ of its present 
mass when $z=1$, whereas with $q=0.2$ we go from $0.87 \widetilde{\mathcal{M}}_{v}$ at $z=1$ to the present $(z=0)$ rest-mass value $\widetilde{\mathcal{M}}_{v}$.

There is support for this kind of galaxy mass evolution as Carlberg et al. (2000) showed that in the CNOC2 and in the Caltech Faint Galaxy Survey galaxies of mass within an order of magnitude of $\mathcal{M}_{*}$ will grow by about $0.15 \mathcal{M}_{*}$ from $z=1$ to $z=0$. Here $\mathcal{M}_{*}$ is the typical mass of a galaxy with luminosity given by the luminosity scale parameter $L_{*}$ of Schechter's LF (eq. 2). Simulations carried out by Murali et al. (2001) agree with Carlberg et al. (2000). Both studies emphasize, however, that these results only pertain to galaxies which are large, that is, with masses near $\mathcal{M}_{*}$, leaving out low mass galaxies which should also grow by mergers and accretion, but not as much as the larger galaxies. Both studies do not mention possible dependency of galaxy mass evolution with morphological types. Therefore, a mild growth such as setting $q=0.2$ for all galaxy morphologies seems a quite reasonable first order approximation considering all these uncertainties.

At this point one must look again at equation (11) to notice that if we replace $\mathcal{M}_{v}$ by $\left[f(z) \widetilde{\mathcal{M}}_{v}\right]$ the mass evolution estimate $f(z)$ is canceled out in that equation. This means that we can use the values of the typical masses at rest with us in equation (11), since, in that expression, the following approximation holds: $\mathcal{M}_{v} \simeq \widetilde{\mathcal{M}}_{v}$. In other words, the observed differential number counts (11) constructed from LF parameters, which is the basic quantity to be used in all analyses of this paper, is, therefore, not affected by galaxy mass evolution, at least to first-order approximation.

Notice that although galaxy mass evolution does not directly change equation (11), it appears indirectly since the selection functions in equation (11) were obtained from LF parameters whose derivation usually include some sort of source evolution.

\subsection{Einstein-de Sitter Cosmology}

We now specialize the equations above to Einstein-de Sitter (EdS) cosmology. This is done by finding the equations for the affine parameter and the area distance in terms of the redshift. It is simple to show that both equations can be written (RS03, §4.2)

$$
\begin{gathered}
\frac{d y}{d z}=\frac{c}{H_{0}(1+z)^{7 / 2}}, \\
d_{A}=\frac{2 c}{H_{0}} \frac{(1+z-\sqrt{1+z})}{(1+z)^{2}},
\end{gathered}
$$

where $H_{0}$ is the Hubble constant and $c$ is the light speed. Throughout this paper we take $H_{0}=100 \mathrm{~km} \mathrm{~s}^{-1} \mathrm{Mpc}^{-1}$ as this is the value adopted by Lin et al. (1999). We aim here to 
factor the cosmology out of LF data and since the value of the Hubble constant is embedded in LF calculations, we must adopt the same value originally set for the LF calculation. In the case of this paper it then must be the same as used by Lin et al. (1999).

An important issue in LF calculations is the volume definition. Currently observers adopt the comoving volume, whereas all equations above assume the proper volume. So, we need a volume conversion, obviously dependent on the cosmological model, in order to actually use the selection function in our equations above. Thus, in the EdS model the selection functions in both these volumes are related by (RS03, footnote 6 and $\S 4.3$ ),

$$
{ }^{\mathrm{PR}} \psi_{v}^{W}=\frac{9}{4}\left(H_{0}\right)^{2}(1+z)^{3}{ }^{\mathrm{C}} \psi_{v}^{W}
$$

where the left superscripts PR and C denote that the selection function is evaluated with the proper and comoving volumes, respectively.

Bearing in mind the results above and spherical symmetry, we are now in position to write the observed differential number counts (11) in terms of the selection function in the EdS cosmological model as follows,

$$
\left[\frac{d N}{d z}\right]_{0}=\left(\frac{36 \pi c^{3}}{H_{0}}\right) \frac{(1+z-\sqrt{1+z})^{2}}{(1+z)^{7 / 2}} \sum_{W} a_{W}\left[\frac{\sum_{v} P_{v}^{W} \mathcal{M}_{v}{ }^{\mathrm{C}} \psi_{v}^{W}}{\sum_{v} P_{v}^{W} \mathcal{M}_{v}}\right]
$$

\section{4. $\mathrm{dN} / \mathrm{dz}$ from LF Data of CNOC2 Galaxy Survey}

The CNOC2 sample was obtained in three wave band filters: $R_{c}, U, B_{A B}$ (hereafter, we shall for notational ease, abbreviate the latter as "B"). This gives us the possible values for the wave band index $W=R_{c}, U, B$. So, according to equation (6) we have $a_{W}=b_{W}$ and since all galaxies appear in all bandwidths, we have that

$$
b_{R_{C}}=b_{B}=b_{U}=1 / 3 .
$$

This catalogue has morphological indexes $v=1,2,3$ denoting the three morphological types in which the sample population was divided, early, intermediate and late spectral types, respectively (Lin et al. 1999). These galaxy morphological types have very approximate dynamical average rest masses equal to $\mathcal{M}_{1}=0.5 \times 10^{11} \mathcal{M}_{\odot}, \mathcal{M}_{2}=0.3 \times 10^{11} \mathcal{M}_{\odot}$ and $\mathcal{M}_{3}=0.1 \times 10^{11} \mathcal{M}_{\odot}($ Sparke and Gallagher 2000, pp. 204 and 264). As discussed in RS03, the CNOC2 sample is such that the proportion $P_{v}$ of each galaxy type population is as follows (RS03, §3.4, tables 1-3),

$$
\left\{\begin{array}{l}
P_{1}^{R_{c}}=P_{1}^{U}=P_{1}^{B}=0.29 \\
P_{2}^{R_{c}}=P_{2}^{U}=P_{2}^{B}=0.24 \\
P_{3}^{R_{c}}=P_{3}^{U}=P_{3}^{B}=0.47
\end{array}\right.
$$


With these results equation (15) can be applied to the CNOC2 sample, yielding,

$$
\left[\frac{d N}{d z}\right]_{0}=\left(\frac{36 \pi c^{3}}{H_{0}{ }^{3}}\right) \frac{(1+z-\sqrt{1+z})^{2}}{(1+z)^{7 / 2}} \sum_{\substack{W=1 \\\left(R_{c}, U, B\right)}}^{3} b_{W}\left[\frac{\sum_{v=1}^{3} P_{v}^{W} \mathcal{M}_{v}\left(\underset{\mathrm{CNOC} 2}{\mathrm{C}} \psi_{v}^{W}\right)}{\sum_{v=1}^{3} P_{v}^{W} \mathcal{M}_{v}}\right]
$$

where the correction

$$
\left({ }_{\mathrm{CNOC} 2}^{\mathrm{C}} \psi_{v}^{W}\right)=\left(H_{0}\right)^{2}\left({ }^{\mathrm{C}} \psi_{v}^{W}\right)
$$

is necessary in order to have correct units. Considering now the CNOC2 figures above, this equation can still be changed to,

$$
\begin{aligned}
{\left[\frac{d N}{d z}\right]_{0}=} & \left(\frac{36 \pi c^{3}}{H_{0}^{3}}\right) \frac{(1+z-\sqrt{1+z})^{2}}{(1+z)^{7 / 2}} \frac{1}{7.92 \times 10^{10}} \times \\
& \times\left[\left(\psi_{1}^{R_{c}}+\psi_{1}^{U}+\psi_{1}^{B}\right) 1.45 \times 10^{10}+\right. \\
& +\left(\psi_{2}^{R_{c}}+\psi_{2}^{U}+\psi_{2}^{B}\right) 7.2 \times 10^{9}+ \\
& \left.+\left(\psi_{3}^{R_{c}}+\psi_{3}^{U}+\psi_{3}^{B}\right) 4.7 \times 10^{9}\right]
\end{aligned}
$$

where the $\mathrm{C}$ and $\mathrm{CNOC} 2$ indexes of the selection function in equation (16) were dropped. The observed differential number count of the CNOC2 redshift survey can then be finally calculated. Tables 1, 2 and 3 below reproduce the numbers calculated and presented in RS03 with errors calculated here (see $\S \mathrm{A}$ ), which are enough to evaluate equation (17). The results are shown in table 4.

Notice that the value of the Hubble constant in equation (17) must be the same as originally used by Lin et al. (1999) in deriving the CNOC2 LF, that is, $H_{0}=100 \mathrm{~km} \mathrm{~s}^{-1} \mathrm{Mpc}^{-1}$.

\section{Differential Density Statistics with CNOC2 Derived Data}

We shall now present the differential density radial statistics required to analyze the galaxy number density distribution and its conceptual implications. Some of this was previously discussed in Ribeiro $(2001,2005)$ and, therefore, $\S 3.1$ is in part a review. Subsequently we will apply these general expressions to the $\mathrm{CNOC} 2$ data obtained in the previous section in two different standard cosmological models. 


\subsection{Basic Equations and Concepts}

\subsubsection{Definitions}

The differential density $\gamma$ at a certain observed distance $d_{0}$ is defined by the following expression,

$$
\gamma=\frac{1}{S_{0}} \frac{d N}{d\left(d_{0}\right)},
$$

where

$$
S_{0}=4 \pi\left(d_{0}\right)^{2}
$$

is the area of the observed shell of radius $d_{0}$. This definition appears to have been initially used in the context of the galaxy distribution by Wertz $(1970,1971)$. As can be easily seen, $\gamma$ is really very much like the similar differential number counts parameter $d N / d z$ (see eq. 4 ), except that it is differential with respect to an observable distance down our past light cone, instead of with respect to $z$ (which is also an observable parameter down our past light cone). Furthermore, it is "normalized" with respect to the "observed surface area" $S_{0}$. Essentially, then, $\gamma$ tells us the rate of growth in the number counts, or more exactly in their density, as one moves down the past light cone in the observable distance $d_{0}$. As is obvious, the behavior of this parameter will depend heavily on which distance we employ for $d_{0}$ - observer area distance, galaxy area distance, luminosity distance, redshift distance, or something else.

Furthermore, Ribeiro (2005) has advanced an extension of $\gamma$ called the integral differential density $\gamma^{*}$, which is simply the integration of $\gamma$ over the observed volume $V_{0}$. Such a definition yields,

$$
\gamma^{*}=\frac{1}{V_{0}} \int_{V_{0}} \gamma d V_{0},
$$

where

$$
V_{0}=\frac{4}{3} \pi\left(d_{0}\right)^{3} .
$$

We should carefully note that $d V_{0}$ is different from the volume element given in the usual form of the metric. This is because it involves $d\left(d_{0}\right)$ down our past light cone, instead of something like $a(t) d r$ at one given time $t=t_{1}$ - that is on space-like surface, usually one of "almost homogeneity." 
It is more useful to write $\gamma$ and $\gamma^{*}$ in terms of the redshift, as follows, ${ }^{5}$

$$
\begin{aligned}
& \gamma(z)=\frac{d N}{d z}\left[S_{0}(z) \frac{d}{d z}\left[d_{0}(z)\right]\right]^{-1} \\
& \gamma^{*}(z)=\frac{1}{V_{0}(z)} \int_{0}^{z} \gamma(z) \frac{d V_{0}}{d z} d z
\end{aligned}
$$

The equations above can be obtained from any given cosmological model, since all quantities on the right hand side can be calculated from the geometry of the model and the assumed matter distribution. The equivalent equations built from data derived galaxy samples should then read as follows,

$$
\begin{gathered}
\gamma_{0}(z)=\left[\frac{d N}{d z}\right]_{0}\left[S_{0} \frac{d}{d z}\left(d_{0}\right)\right]^{-1} \\
\gamma_{0}^{*}(z)=\frac{1}{V_{0}} \int_{0}^{z} \gamma_{0} \frac{d V_{0}}{d z} d z
\end{gathered}
$$

Notice that by their definitions in equations (18), (19), (20), (21), the following result holds,

$$
\gamma^{*}=\frac{N}{V_{0}}=\langle n\rangle
$$

where $\langle n\rangle$ is the average number density.

\subsubsection{Conceptual Implications}

\section{Spatial and Observational Homogeneities}

In order to interpret the meaning and significance of $\gamma$ and $\gamma^{*}$ further, we emphasize that in cosmological models we can define two distinct types of homogeneity. Spatial homogeneity is defined in the usual sense, that is, the usual spatial density $\rho$ appearing on the right hand side of Einstein's field equations is dependent only on time, $\rho=\rho(t)$, which means that in each constant time hypersurface the local density remains unchanged. So, spatial homogeneity means that quantities such as density are constant on a space-like foliation of surfaces. The primary example, of course, are the surfaces of homogeneity in FLRW.

\footnotetext{
5 This notation for the differential densities is slightly different than in Ribeiro (2005), since here we follow RS03 very closely and adopt the subscript index zero to refer to observed quantities, built from galaxy sample derived observations as much as possible, whereas the unindexed quantities refer to theoretical expressions, built from theory.
} 
However, there is another type of homogeneity, observational homogeneity, that can also be defined in cosmology. It is the property that some average density calculated with observational data derived from galaxy redshift surveys is constant. This is sometimes what one has in mind when one talks about the possible homogeneity of the galaxy distribution in the context of observational cosmology. So, observational homogeneity is defined in terms of average observational quantities along our past like cone, instead of in a given space-like surface. Operationally, the observational average density $\left\langle\rho_{0}\right\rangle$ is constant. ${ }^{6}$ How to relate $\left\langle\rho_{0}\right\rangle$ to the above-mentioned $\rho(t)$, how to determine $\rho(t)$ from an observed $\left\langle\rho_{0}\right\rangle$, and what the relationship between observational homogeneity and spatial homogeneity are key issues.

Since a basic method for observationally dealing with galaxies is by counting them, it is reasonable to state formally that observational homogeneity is achieved when the average number density $\left\langle n_{0}\right\rangle$ remains constant, as one averages over different volumes down our past light cone. So,

$$
\left\langle n_{0}\right\rangle \equiv \frac{N_{0}}{V_{0}}=\text { constant }
$$

where $N_{0}$ is the number of observed galaxies counted within a certain volume $V_{0}$. As indicated above, this definition of observational density implies that both $N_{0}$ and $V_{0}$ are obtained along our past light cone. So, equation (27) is not applicable over space-like surfaces.

The practical calculation of $\left\langle n_{0}\right\rangle$ by means of equation (27) encounters some basic operational difficulties. We immediately face the problem of identifying the volume $V_{0}$. Actually, since $N_{0}$ is observed, $V_{0}$ should also be an observed quantity. But, we cannot yet translate the observed redshift $z$ into a cosmological distance without a definite cosmological model. So, we must choose a certain distance measure among several possibilities from the model (many workers often choose the comoving distance), observe $N_{0}$ and calculate $\left\langle n_{0}\right\rangle$. Therefore, although spatial homogeneity can be uniquely defined relative to a space-like slicing, observational homogeneity cannot be. Obviously, a model can satisfy spatial homogeneity, without satisfying observational homogeneity, or vice-versa. In fact this will often be the case.

This was shown to be correct in Ribeiro (2001, 2005), with earlier discussions in Ribeiro (1992, 1993, 1995). Since we can also obtain $N$ from theory, Ribeiro (2005) showed that, although EdS cosmology is spatially homogeneous by definition, it may or may not be observationally homogeneous, depending on the particular type of distance chosen. Thus, according to Ribeiro (2005), an FLRW model can satisfy both types of homogeneity, spatial

\footnotetext{
${ }^{6}$ The notation may be a bit confusing here, as $\rho_{0}$ is the local density at the present constant time $t_{0}$, whereas $\left\langle\rho_{0}\right\rangle$ is the average of $\rho[t(r)]$ along the null cone $t(r)$, since $\rho[t(r)]$ will change along the past null cone (see Ribeiro 2001 for in depth discussions of this topic).
} 
and observational, if, and only if, one adopts the galaxy area distance, or comoving distance, in the definition of observational homogeneity, as given by equation (27). Furthermore, it is clear that lack of observational homogeneity is perfectly consistent with spatial homogeneity, and that there will be cases of observational homogeneity in which there is no spatial homogeneity (Rangel Lemos and Ribeiro 2006, in preparation). In specifying the link between the two the distance being used is crucial!

From this point of view, interpreting $\gamma$ and $\gamma^{*}$ is straightforward. Considering equations (26) and (27) it is clear that $\gamma_{0}^{*}=\left\langle n_{0}\right\rangle$. This means that $\gamma$ is a useful tool for determining $\left\langle n_{0}\right\rangle$. But this is true only if we are trying to determine whether or not the galaxy number count $N$ is observationally homogeneous.

\section{Other Remarks}

It is important to point out that only one of the two factors on the right hand side of equation (24), can, at present, be calculated directly from observations. Only the observed differential count $[d N / d z]_{0}$ can be evaluated from equation (11) or one of its specialized forms (15), (16), (17). ${ }^{7}$ To calculate the second factor of equation (24), the geometrical part involving $S_{0}$ and $d_{0}$, one must still assume a cosmological model, inasmuch as obtaining observational distances, either by standard candles or standard rods, of every galaxy in a redshift survey is currently beyond our observational capabilities.

Along with this, as we have already indicated above and in the introduction, there is the key problem that the observational distance $d_{0}$ as obtained from theory is not unique, meaning that the observed density of the galaxy distribution is dependent on this distance choice. This applies even both to $\gamma$ and to $\gamma^{*}-\gamma^{*}$ depends on the volume $V_{0}$ over which the galaxies are counted, which in turn depends on the distance choice. We have already seen that that this choice can make a significant difference. Which distance is appropriate?

As it has been extensively argued elsewhere, these are the basic, but, so far, not generally acknowledged, dilemmas underlying the observational determination of the possible smoothness of the universe (Ribeiro 2001). Bearing these points in mind, it becomes clear that comparing $\gamma$ with $\gamma_{0}$ and $\gamma^{*}$ with $\gamma_{0}^{*}$ does not provide a true check of the cosmological model against observations, since the assumed cosmological model appears in both pairs of

\footnotetext{
7 The observed differential number count $[d N / d z]_{0}$ does not need to be estimated only from LF data, as it can be directly observed. Nevertheless, in the context of this paper, we are recovering it from the LF's, since those are often what is made available by the observers.
} 
equations. $^{8}$ We cannot yet determine the geometrical factors purely observationally - they must be calculated from the cosmological model which we adopt on other evidential grounds. Therefore, such a comparison is merely a check of consistency, the best one can do at the moment considering the present limitations in determining the characteristics and distances of galaxies.

\subsubsection{Cosmological Distances and the Reciprocity Law}

As stated above the observed distance $d_{0}$ is not unique. There are at least four distinct possibilities (Ellis 1971; Ribeiro 2001, 2002, 2005): the observer area distance $d_{A}$ (which is equal to the angular diameter distance), already introduced in $§ 2.1$ above, the luminosity distance $d_{L}$, the galaxy area distance $d_{G}$ (also known as effective distance, angular size distance, transverse comoving distance or proper motion distance) and the redshift distance $d_{z}$. These distances have expressions dependent on the assumed cosmological model. In FLRW cosmologies the last one may be written as,

$$
d_{z}=\frac{c z}{H_{0}}
$$

The other three distances are linked to each other by the remarkable reciprocity theorem or Etherington's reciprocity law, valid for any cosmological model and which reads as follows (Etherington 1933; Ellis 1971; Plebański and Krasiński 2006, p. 256),

$$
d_{L}=(1+z)^{2} d_{A}=(1+z) d_{G} .
$$

These distances can, in principle, be observationally determined, since this law requires that source and observer should only be connected by null geodesics (Ellis 1971). Furthermore, if we assume a cosmological model, theoretically defined distances such as the comoving distance, the proper distance, the distance defined by Mattig's formula, etc, can be reduced to one of the four above (see, e.g., Ribeiro 2001, 2005).

\footnotetext{
8 This is also true for any density constructed with galaxy samples (Ribeiro 2001; Abdalla, Mohayaee and Ribeiro 2001).
} 


\subsection{Einstein-de Sitter Model}

In EdS, besides equation (28) the other observational distances are straightforwardly calculated in terms of $z$ as being given by (Ribeiro 2001, 2005),

$$
\begin{aligned}
d_{L} & =\frac{2 c}{H_{0}}(1+z-\sqrt{1+z}), \\
d_{A} & =\frac{2 c}{H_{0}} \frac{(1+z-\sqrt{1+z})}{(1+z)^{2}}, \\
d_{G} & =\frac{2 c}{H_{0}}\left(\frac{1+z-\sqrt{1+z}}{1+z}\right),
\end{aligned}
$$

and $^{9}$ these results allow us to derive expressions for the observed area and volume for each distance, i.e., $S_{L}, S_{A}, S_{G}, S_{z}$ and $V_{L}, V_{A}, V_{G}, V_{z}$ in terms of the redshift (Ribeiro 2005). Thus we are able to use the results of table 4 to calculate the observed differential density (24) and, after numerical interpolations and quadratures, the observed integral differential density (25).

Table 5 shows the results for both differential densities in each observational distance, as well as the values for each distance in the redshift range of the CNOC2 sample. The figures show clearly that the general conclusions about the dependence on the distance choice in measuring the density of the distribution of galaxies hold. Both the observed differential density $\gamma_{0}$ and the integral differential density $\gamma_{0}^{*}$ increase for higher $z$ when written in terms of the area distance $d_{A}$, whereas both decrease when they are written in terms of $d_{L}$ or $d_{z}$. There is a slight increase when $d_{G}$ is used, but that is mostly within the error margins and, even so, so small for both $\left[\gamma_{G}\right]_{0}$ and $\left[\gamma_{G}^{*}\right]_{0}$ that one can consider that in practice both density statistics remain unchanged when written as functions of the galaxy area distance $d_{G}$. Since this last distance is the same as the comoving distance (Ribeiro 2005), widely used in testing both the possible homogeneity or inhomogeneity of the galaxy distribution, it seems that the conclusion reached in Ribeiro (2001) and (2005) about $d_{G}$ being inappropriate for measuring the possible inhomogeneity of the Universe do seem to hold, at least as far as the CNOC2 survey is concerned. Comoving distance is only suitable to test spatial homogeneity in FLRW models. These results are shown graphically in figures 1 and 2 .

Figures 3 and 4 show both differential densities obtained from the CNOC2 data and plotted against the luminosity and redshift distances, respectively. $\gamma$ and $\gamma^{*}$ decrease as $z$ increases, showing a tendency towards a power law behavior at scales of $z>0.1$. Since power

\footnotetext{
${ }^{9}$ In EdS model the comoving distance is equal to $d_{G}$, apart from a constant (Ribeiro 2005).
} 
laws are usually related to self-similar patterns (Pietronero 1987; Ribeiro and Miguelote 1998; Gabrielli et al. 2005), we have drawn straight lines for reference, whose slopes would approximately give the respective fractal dimensions of the distribution. The plots themselves do not prove that the CNOC2 galaxy distribution is fractal for $z>0.1$, but does not entirely rule out this possibility. In our view, this possibility clearly deserves further investigation.

Of course, this is not saying that the spatial distribution of galaxies may be fractal. That is fitted by an EdS, or other FLRW, model and therefore approximately homogeneous on large scales. What it is saying is that the observationally determined average density of galaxies down our past light cone is possibly fractal.

\subsection{FLRW Cosmology with $\Omega_{\mathrm{m}_{0}}=0.3$ and $\Omega_{\Lambda_{0}}=0.7$}

The differential densities can only be obtained in this cosmological model by means of numerical integrations. Here we shall not provide details of these calculations, which are the subject of a forthcoming paper (Iribarrem, Ribeiro and Stoeger 2006, in preparation), but limit ourselves in presenting data and plots containing similar results as in the EdS case. Suffice it to say that the methodology is precisely the same as described in $\S 3.2$ : take the data in table 4 and apply them to equations (24) and (25), but evaluating numerically the geometrical part involving $d_{0}, S_{0}$ and $V_{0}$ in this FLRW cosmology. The results for all differential densities and distances are shown in table 6.

Figures 5 and 6 show both differential densities against the redshift using the theoretical differential number count $d N / d z$ obtained with the model, whereas figures 7 and 8 replace $d N / d z$ with the observed $[d N / d z]_{0}$ of table 4 obtained from the CNOC2 survey. One can clearly notice that all densities are strongly dependent on the distance choice, not only the theoretical, but the observed ones. However, the densities calculated with the galaxy area distance $d_{G}$ change from a small decrease in the EdS model to a more pronounced one in figures 7 and 8 . That decrease does not happen in the theoretical plots shown in figures 5 and 6 , meaning that it can only be attributed to the $\mathrm{CNOC} 2$ data. One can also notice a clear power law pattern for densities plotted with the luminosity and redshift distances $d_{L}$ and $d_{z}$ at ranges where the redshift is higher than 0.1.

Finally, figures 9 and 10 show both differential densities respectively plotted against $d_{L}$ and $d_{z}$. As in the EdS model, there is a power law pattern in the tail of the plot, that is, for $z>0.1$. That pattern seems to be even more linear than for the EdS cosmology. Straight lines for reference have also been drawn, showing the approximate slope of the linear decaying 
behavior. Since power law patterns are usually related to self-similar fractal structures, the possibility that some standard cosmological models possess observational fractal patterns at ranges of $z>0.1$ deserves further investigation in other galaxy survey samples.

\section{Conclusions}

In this paper we have extended the theory developed by Ribeiro and Stoeger (2003) for linking the relativistic cosmology source count to the luminosity function (LF) in order to extract the differential number count $d N / d z$ from LF data. Since LF parameters assume a cosmological model, we developed the analytical means to factor out the assumed cosmology from the LF parameters, while preserving the LF analytical assumptions and source evolution corrections. We have applied this methodology to the CNOC2 galaxy redshift survey (Lin et al. 1999) and successfully extracted the observed differential number count $[d N / d z]_{0}$ from this galaxy catalogue. Then we used this extracted data to carry out a statistical analysis based on the radial differential densities discussed in Ribeiro (2005). We have done so in the Einstein-de Sitter cosmology and the currently favored $\Omega_{m_{0}}=0.3, \Omega_{\Lambda_{0}}=0.7$ standard cosmological model. Our main aim was to test Ribeiro's (2005) proposition that different distance definitions when applied to galaxy survey analyses introduce ambiguities that render the statistical results unavoidably dependent on the distance choice.

Our results show that Ribeiro's (2005) proposition is sound for both models under study. We calculated two types of radial statistical tools, namely the differential density $\gamma$ and the integral differential density $\gamma^{*}$, with the CNOC2 derived data and found out that both have a strong dependency on the distance choice, as well as, showing an observational power law pattern for $z>0.1$ when $\gamma$ and $\gamma^{*}$ are calculated with the luminosity distance $d_{L}$ and the redshift distance $d_{z} \cdot{ }^{10}$ This power law decay is present in both cosmological models, EdS and $\Omega_{m_{0}}=0.3, \Omega_{\Lambda_{0}}=0.7$ standard model, although the linear power law tendency is more pronounced in the latter cosmology. If we cautiously interpret this power law pattern as possible evidence of a self-similar fractal structure, that would mean observational galaxy distribution fractal dimensions in the range $D \approx 1.6-1.8$ at scales of $z>0.1$.

In addition to the remarks of $\S 3.1 .2$ above, it is helpful to remember that the cumulative number counts $N_{0}$ is a dimensionless observational quantity with no dependence on

\footnotetext{
10 Notice that this power law pattern only appears with some observational quantities. As stressed in $\S 3.1 .2$ above, the results of this paper only refer to observational homogeneity as the underlying models still keep their spatial homogeneity, which is there by construction. See also Ribeiro (2001) for in depth discussions of this topic.
} 
any volume definition. It can be used in other contexts, like star counting, in a purely observational setting, having no relation to theory. However, in determining related parameters $-\left\langle n_{0}\right\rangle$ for example - we must know over what volume $N_{0}$ has been counted, and this involves determining or assuming a definite cosmological model. In a cosmological context the theoretical counterpart of $N_{0}$, that is $N$, scales with the redshift according to the overall mass-energy distribution of the universe, and this theoretical dependence given by the cosmological models is what we seek to relate to number count observations, that is, to $N_{0}$.

The link between $N$ and $N_{0}$, or between derivative differential number counts $d N / d z$ and $[d N / d z]_{0}$, is provided by equation (1). In this equation $d N$ is written in terms of the proper number density $n$. Since in equation (1) $n$ is written in terms of proper volumes, it was necessary to use the area distance - to tie together the locally observed cross-sectional area, which enters in the proper volume, with the observed solid angle. So, we can only use equation (1) to obtain $d N / d z$ from a number density if we write $n$ in terms of the proper density. If $n$ is not given in terms of the proper volume, a conversion is required, that being the case when one deals with the LF in its usual form. In theoretical calculations we can take the local mass-energy density $\rho$ that appears on the right hand side of Einstein's field equation and get $n$ from it (RS03, eq. 16). Then $N=N(z)$ is independent of any volume, but still dependent on the overall matter distribution in the universe, which, through General Relativity, is given by the spacetime geometry. In particular, the affine parameter $y$ is required in deriving $N$ and the dependence of $y$ on $z$ is given by the cosmology specifically adopted (e.g., eq. 12 above).

As a result of the procedure outlined above, the final $d N / d z$ is dimensionless and volume independent, meaning that we are then free to replace $d N / d z$ as given by equation (1) with our observed, and also dimensionless, $[d N / d z]_{0}$ in order to define other number densities in different volumes. The $\gamma_{0}$ and $\gamma_{0}^{*}$ functions are just different number densities, built in such a way as to show not only the scaling with $z$ (dependence with the overall matter distribution), but also its dependence on one of the possible volume definitions of the assumed cosmological model (geometrical dependence). The idea is to make explicit the geometrical dependence on distance choice, whereas this choice has been implicit in most discussions about the large-scale galaxy distribution in the Universe.

We should also mention that linking the observational counts and densities with spatial ones, and with spatial homogeneity, takes some work. One way one can do this is by using $[d N / d z]_{0}$ data and Ellis' formula (1), or its observational version (eq. 4) (Stoeger 1987; Araújo and Stoeger 1999) to obtain $n(z)$ at different redshifts, by observationally determining $d_{A}=d_{A}(z)$ for at least some galaxies in the sample, and see if this $n$ as function of $z$ agrees with the FLRW theoretical results. This would really give us the proper densities on space- 
like slices, and enable us to see spatial homogeneity. This is quite different from integrating over large volumes down our past light cone to get observationally averaged densities, which convolve proper densities from a wide range of redshifts.

As a final remark, since spatial and observational homogeneities are two different features of a cosmological model, as extensively discussed in $§ 3.1 .2$ above, it should be clear by now that further work needs to be done in this area, including a clear treatment of how one observationally tests for spatial homogeneity using observed galaxy distributions and other data, without first demonstrating or assuming that the cosmological region under consideration is "almost FLRW."

We thank the referee for very useful remarks and suggestions which improved the paper. Two of us (V.V.L.A. and M.B.R.) are grateful to CNPq and FAPERJ for financial support.

\section{A. Error Analysis}

In RS03 the selection function for Schechter's type LF was written in terms of the absolute magnitude $M_{W}$ in the filter $W$ as follows (RS03, eq. 96),

$$
\psi^{W}(z)=\int_{-\infty}^{M_{W}(z)}(0.4 \ln 10) \phi_{*} 10^{0.4(1+\alpha)\left(M_{*}-\bar{M}_{W}\right)} \exp \left[-10^{0.4\left(M_{*}-\bar{M}_{W}\right)}\right] d \bar{M}_{W},
$$

where the three parameters that characterize the LF are the space density of galaxies $\phi_{*}$, the asymptotic slope of the faint end $\alpha$, and the luminosity scale given in terms of absolute magnitude $M_{*}$. The CNOC2 survey incorporates a luminosity scale evolution such that $M_{*}$ was rewritten in terms of two other parameters $M_{*}^{\prime}$ and $Q$ (Lin et al. 1999, eq. 10; RS03, eq. 63) leading equation (A1) to be rewritten as follows,

$$
\begin{aligned}
\psi^{W}(z) & =0.4 \ln 10 \phi_{*} \\
& \times \int_{-\infty}^{M_{W}(z)} 10^{0.4(1+\alpha)\left[M_{*}^{\prime}-Q(z-0.3)-\bar{M}_{W}\right]} \exp \left\{-10^{0.4\left[M_{*}^{\prime}-Q(z-0.3)-\bar{M}_{W}\right]}\right\} d \bar{M}_{W} .
\end{aligned}
$$

Lin et al. (1999) provided uncertainties for these four parameters, $M_{*}^{\prime}, \alpha, \phi_{*}, Q$ in their tables 1-3. These errors were propagated in equation (A2) by means of standard error propagation techniques in order to produce the uncertainties appearing in tables 1, 2, 3 of this paper. Numerical and algebraic computing scripts were used for this task.

To obtain uncertainties for the observed differential number counts $[d N / d z]_{0}$ shown in table 4, we have used equation (17) to further propagate the already calculated errors for the 
selection functions. Similarly, by means of equation (22) it was straightforward to evaluate uncertainties for the differential density $\gamma$. These results are shown in tables 5 and 6 .

To evaluate uncertainties for the integral differential density $\gamma^{*}$ we proceeded as follows. From equation (26) it is clear that $\delta \gamma^{*}=\delta N / V$. Since we can write $N=\int(d N / d z) d z$, it is easy to find $\delta N$, and, therefore, uncertainties of the integral differential density yield,

$$
\delta \gamma^{*}=\left(\frac{1}{V}\right)\left(\frac{d^{2} N}{d z^{2}}\right)^{-1}\left(\frac{d N}{d z}\right) \delta(d N / d z) .
$$

Equation (17) and the already tabulated errors of the observed differential number counts $\delta[d N / d z]_{0}$ allowed us to obtain the values for $\delta \gamma_{0}^{*}$. The results are shown in tables 5 and 6 .

\section{REFERENCES}

Abdalla, E., Mohayaee, R. and Ribeiro, M. B. 2001, Fractals, 9, 451, astro-ph/9910003

Araújo, M. E. and Stoeger, W. R. 1999, Phys. Rev. D 60, 104020

Carlberg, R. G. et al. 2000, ApJ, 532, L1, astro-ph/0002036

Ellis, G. F. R. 1971, General Relativity and Cosmology, Proc. Int. School Phys. "Enrico Fermi", R. K. Sachs, New York: Academic Press, 104

Etherington, I. M. H. 1933, Phil. Mag., 15, 761

Gabrielli, A., Sylos-Labini, F., Joyce, M., and Pietronero, L. 2005, Statistical Physics for Cosmic Structures, Berlin: Springer

Hogg, D. W., Eisenstein, D. J., Blanton, M. R., Bahcall, N. A., Brinkman, J., Gunn, J. E., and Schneider, D. P. 2005, ApJ, 624, 54

Lin, H. et al. 1999 (CNOC2 Survey), ApJ, 518, 533, astro-ph/9902249

Murali, C. et al. 2001, astro-ph/0106282

Pebbles, P. J. E. 1993, Principles of Physical Cosmology (Princeton, N. J.: Princeton University Press), pp. 24-45, 209-224, 457-468.

Plebański, J., and Krasiński, A. 2006, An Introduction to General Relativity and Cosmology (Cambridge University Press)

Pietronero, L. 1987, Physica A, 144, 257 
Ribeiro, M. B. 1992, ApJ, 395, 29

Ribeiro, M. B. 1993, ApJ, 415, 469

Ribeiro, M. B. 1995, ApJ, 441, 477, astro-ph/9910145

Ribeiro, M. B. 2001, Gen. Rel. Grav., 33, 1699, astro-ph/0104181

Ribeiro, M. B. 2002, Observatory, 122, 201, gr-qc/9910014

Ribeiro, M. B. 2005, Astron. Astrophys., 429, 65, astro-ph/0408316

Ribeiro, M. B., and Miguelote, A. Y. 1998, Brazilian J. Phys., 28, 132, astro-ph/9803218

Ribeiro, M. B., and Stoeger, W. R. 2003 (RS03), ApJ, 592, 1, astro-ph/0304094

Sparke, L. S., and Gallagher, J. S. 2000, Galaxies in the Universe, Cambridge University Press

Stoeger, W. R. 1987, "Almost FLRW Observational Cosmologies," in Theory and Observational Limits in Cosmology, W. R. Stoeger, editor, Specola Vaticana (Vatican Observatory), pp. 275-305.

Wertz, J. R. 1970, Newtonian Hierarchical Cosmology, Ph.D. Thesis, University of Texas at Austin

Wertz, J. R. 1971, ApJ, 164, 227 
Table 1. CNOC2 selection function ${ }^{\mathrm{a}}$ results from RS03 plus errors evaluated here (see appendix $\mathrm{A}$ ) in the $R_{c}$ band vs. redshift.

\begin{tabular}{cccc}
\hline \hline$z$ & $\psi_{1}^{R_{c}}$ & $\psi_{2}^{R_{c}}$ & $\psi_{3}^{R_{c}}$ \\
\hline 0.05 & $0.0181 \pm 0.0038$ & $0.0120 \pm 0.0040$ & $0.0220 \pm 0.0130$ \\
0.12 & $0.0182 \pm 0.0038$ & $0.0121 \pm 0.0041$ & $0.0221 \pm 0.0130$ \\
0.25 & $0.0183 \pm 0.0039$ & $0.0123 \pm 0.0041$ & $0.0223 \pm 0.0130$ \\
0.4 & $0.0185 \pm 0.0039$ & $0.0125 \pm 0.0042$ & $0.0225 \pm 0.0132$ \\
0.55 & $0.0186 \pm 0.0040$ & $0.0127 \pm 0.0044$ & $0.0227 \pm 0.0135$ \\
0.75 & $0.0187 \pm 0.0040$ & $0.0130 \pm 0.0045$ & $0.0230 \pm 0.0141$ \\
0.9 & $0.0187 \pm 0.0041$ & $0.0132 \pm 0.0046$ & $0.0232 \pm 0.0148$ \\
1.0 & $0.0187 \pm 0.0041$ & $0.0133 \pm 0.0047$ & $0.0234 \pm 0.0153$ \\
\hline
\end{tabular}

aSubscript numbers denote the spectral type morphology adopted for the CNOC2 survey (see $\S 2.4$ above). Units are $\mathrm{Mpc}^{-3}$. 
Table 2. CNOC2 selection function results from RS03 plus errors evaluated here (see appendix A) in the $U$ band vs. redshift.

\begin{tabular}{cccc}
\hline \hline$z$ & $\psi_{1}^{U}$ & $\psi_{2}^{U}$ & $\psi_{3}^{U}$ \\
\hline 0.05 & $0.0180 \pm 0.0031$ & $0.0119 \pm 0.0038$ & $0.0284 \pm 0.0134$ \\
0.12 & $0.0182 \pm 0.0031$ & $0.0121 \pm 0.0038$ & $0.0289 \pm 0.0135$ \\
0.25 & $0.0186 \pm 0.0032$ & $0.0123 \pm 0.0039$ & $0.0297 \pm 0.0139$ \\
0.4 & $0.0189 \pm 0.0033$ & $0.0126 \pm 0.0040$ & $0.0307 \pm 0.0145$ \\
0.55 & $0.0192 \pm 0.0033$ & $0.0128 \pm 0.0042$ & $0.0317 \pm 0.0152$ \\
0.75 & $0.0194 \pm 0.0034$ & $0.0131 \pm 0.0043$ & $0.0330 \pm 0.0162$ \\
0.9 & $0.0195 \pm 0.0034$ & $0.0134 \pm 0.0045$ & $0.0341 \pm 0.0172$ \\
1.0 & $0.0196 \pm 0.0034$ & $0.0135 \pm 0.0045$ & $0.0348 \pm 0.0179$ \\
\hline
\end{tabular}


Table 3. CNOC2 selection function results from RS03 plus errors evaluated here (see appendix A) in the $B_{A B}$ band vs. redshift.

\begin{tabular}{cccc}
\hline \hline$z$ & $\psi_{1}^{B}$ & $\psi_{2}^{B}$ & $\psi_{3}^{B}$ \\
\hline 0.05 & $0.0182 \pm 0.0033$ & $0.0122 \pm 0.0036$ & $0.0252 \pm 0.0130$ \\
0.12 & $0.0183 \pm 0.0033$ & $0.0123 \pm 0.0036$ & $0.0254 \pm 0.0130$ \\
0.25 & $0.0185 \pm 0.0034$ & $0.0125 \pm 0.0037$ & $0.0257 \pm 0.0131$ \\
0.4 & $0.0187 \pm 0.0034$ & $0.0128 \pm 0.0038$ & $0.0260 \pm 0.0133$ \\
0.55 & $0.0189 \pm 0.0035$ & $0.0130 \pm 0.0040$ & $0.0264 \pm 0.0136$ \\
0.75 & $0.0190 \pm 0.0035$ & $0.0133 \pm 0.0041$ & $0.0268 \pm 0.0143$ \\
0.9 & $0.0191 \pm 0.0035$ & $0.0135 \pm 0.0042$ & $0.0272 \pm 0.0150$ \\
1.0 & $0.0191 \pm 0.0035$ & $0.0136 \pm 0.0043$ & $0.0274 \pm 0.0155$ \\
\hline
\end{tabular}


Table 4. CNOC2 differential counts for all wave bands and morphologies vs. redshift.

\begin{tabular}{cc}
\hline \hline$z$ & {$\left[\frac{d N}{d z}\right]_{0}$} \\
\hline 0.05 & $(2.914 \pm 1.024) \times 10^{7}$ \\
0.12 & $(1.394 \pm 0.491) \times 10^{8}$ \\
0.25 & $(4.409 \pm 1.564) \times 10^{8}$ \\
0.4 & $(8.125 \pm 2.920) \times 10^{8}$ \\
0.55 & $(1.142 \pm 0.417) \times 10^{9}$ \\
0.75 & $(1.487 \pm 0.555) \times 10^{9}$ \\
0.9 & $(1.679 \pm 0.639) \times 10^{9}$ \\
1.0 & $(1.780 \pm 0.686) \times 10^{9}$ \\
\hline
\end{tabular}


Table 5. Redshifts, distances and differential densities calculated with CNOC2 differential number count data in an Einstein-de Sitter cosmology. Distances are in Mpc, densities in $\mathrm{Mpc}^{-3}$ and $H_{0}=100 \mathrm{~km} \mathrm{~s}^{-1} \mathrm{Mpc}^{-1}$. The differential densities were calculated using equations (24) and (25), where the observed differential number count $[d N / d z]_{0}$ was given by the survey data results shown in table 4 , whereas the second factor in the right hand side of equation (24) was obtained theoretically, that is, from the assumed cosmological model.

\begin{tabular}{|c|c|c|c|c|c|c|c|c|c|c|c|c|}
\hline$z$ & $d_{A}$ & $d_{G}$ & $d_{L}$ & $d_{z}$ & {$\left[\gamma_{A}\right]_{0}$} & {$\left[\gamma_{G}\right]_{0}$} & {$\left[\gamma_{L}\right]_{0}$} & {$\left[\gamma_{z}\right]_{0}$} & {$\left[\gamma_{A}^{*}\right]_{0}$} & {$\left[\gamma_{G}^{*}\right]_{0}$} & {$\left[\gamma_{L}^{*}\right]_{0}$} & {$\left[\gamma_{z}^{*}\right]_{0}$} \\
\hline 0.05 & 138 & 144 & 152 & 150 & $0.0485 \pm 0.0050$ & $0.0399 \pm 0.0041$ & $0.0328 \pm 0.0034$ & $0.0344 \pm 0.0036$ & $0.0437 \pm 0.0074$ & $0.0377 \pm 0.0064$ & $0.0326 \pm 0.0055$ & $0.0338 \pm 0.0057$ \\
\hline 0.12 & 295 & 330 & 370 & 360 & $0.0640 \pm 0.0066$ & $0.0402 \pm 0.0041$ & $0.0256 \pm 0.0026$ & $0.0286 \pm 0.0029$ & $0.0561 \pm 0.0095$ & $0.0399 \pm 0.0068$ & $0.0284 \pm 0.0048$ & $0.0309 \pm 0.0052$ \\
\hline 0.25 & 506 & 633 & 791 & 749 & $0.1044 \pm 0.0107$ & $0.0408 \pm 0.0042$ & $0.0169 \pm 0.0017$ & $0.0208 \pm 0.0021$ & $0.0791 \pm 0.0147$ & $0.0405 \pm 0.0075$ & $0.0207 \pm 0.0038$ & $0.0244 \pm 0.0045$ \\
\hline 0.4 & 663 & 928 & 1300 & 1199 & $0.1795 \pm 0.0186$ & $0.0414 \pm 0.0043$ & $0.0111 \pm 0.0011$ & $0.0150 \pm 0.0016$ & $0.1124 \pm 0.0236$ & $0.0410 \pm 0.0086$ & $0.0149 \pm 0.0031$ & $0.0190 \pm 0.0040$ \\
\hline 0.55 & 761 & 1180 & 1829 & 1649 & $0.3067 \pm 0.0322$ & $0.0420 \pm 0.0044$ & $0.0076 \pm 0.0008$ & $0.0111 \pm 0.0012$ & $0.1540 \pm 0.0370$ & $0.0414 \pm 0.0099$ & $0.0111 \pm 0.0027$ & $0.0152 \pm 0.0036$ \\
\hline 0.75 & 836 & 1463 & 2561 & 2248 & $0.6456 \pm 0.0694$ & $0.0427 \pm 0.0046$ & $0.0048 \pm 0.0005$ & $0.0078 \pm 0.0008$ & $0.2242 \pm 0.0663$ & $0.0418 \pm 0.0124$ & $0.0078 \pm 0.0023$ & $0.0115 \pm 0.0034$ \\
\hline 0.9 & 866 & 1646 & 3127 & 2698 & $1.2154 \pm 0.1339$ & $0.0431 \pm 0.0047$ & $0.0036 \pm 0.0004$ & $0.0061 \pm 0.0007$ & $0.2891 \pm 0.1029$ & $0.0422 \pm 0.0150$ & $0.0061 \pm 0.0022$ & $0.0096 \pm 0.0034$ \\
\hline 1.0 & 878 & 1756 & 3512 & 2998 & $2.0200 \pm 0.2268$ & $0.0433 \pm 0.0049$ & $0.0030 \pm 0.0003$ & $0.0053 \pm 0.0006$ & $0.3387 \pm 0.1398$ & $0.0423 \pm 0.0175$ & $0.0053 \pm 0.0022$ & $0.0085 \pm 0.0035$ \\
\hline
\end{tabular}


Table 6. Redshifts, distances and differential densities calculated with CNOC2 differential number count data in a $\Omega_{m_{0}}=0.3, \Omega_{\Lambda_{0}}=0.7$ standard cosmology. Distances are in Mpc, densities in $\mathrm{Mpc}^{-3}$ and $H_{0}=100 \mathrm{~km} \mathrm{~s}^{-1} \mathrm{Mpc}^{-1}$. The differential densities were calculated similarly as explained in the caption of table 5 .

\begin{tabular}{|c|c|c|c|c|c|c|c|c|c|c|c|c|}
\hline$z$ & $d_{A}$ & $d_{G}$ & $d_{L}$ & $d_{z}$ & {$\left[\gamma_{A}\right]_{0}$} & {$\left[\gamma_{G}\right]_{0}$} & {$\left[\gamma_{L}\right]_{0}$} & {$\left[\gamma_{z}\right]_{0}$} & {$\left[\gamma_{A}^{*}\right]_{0}$} & {$\left[\gamma_{G}^{*}\right]_{0}$} & {$\left[\gamma_{L}^{*}\right]_{0}$} & {$\left[\gamma_{z}^{*}\right]_{0}$} \\
\hline 0.05 & 141 & 148 & 156 & 150 & $0.0438 \pm 0.0045$ & $0.0360 \pm 0.0037$ & $0.0297 \pm 0.0031$ & $0.0344 \pm 0.0036$ & $0.0405 \pm 0.0069$ & $0.0350 \pm 0.0059$ & $0.0302 \pm 0.0051$ & $0.0338 \pm 0.0057$ \\
\hline 0.12 & 312 & 350 & 392 & 360 & $0.0506 \pm 0.0052$ & $0.0320 \pm 0.0033$ & $0.0205 \pm 0.0021$ & $0.0286 \pm 0.0029$ & $0.0472 \pm 0.0080$ & $0.0336 \pm 0.0057$ & $0.0239 \pm 0.0040$ & $0.0309 \pm 0.0052$ \\
\hline 0.25 & 565 & 706 & 882 & 749 & $0.0662 \pm 0.0068$ & $0.0266 \pm 0.0027$ & $0.0112 \pm 0.0012$ & $0.0208 \pm 0.0021$ & $0.0571 \pm 0.0106$ & $0.0292 \pm 0.0054$ & $0.0150 \pm 0.0028$ & $0.0244 \pm 0.0045$ \\
\hline 0.4 & 776 & 1086 & 1521 & 1199 & $0.0910 \pm 0.0094$ & $0.0226 \pm 0.0023$ & $0.0062 \pm 0.0006$ & $0.0150 \pm 0.0016$ & $0.0702 \pm 0.0147$ & $0.0256 \pm 0.0054$ & $0.0093 \pm 0.0020$ & $0.0190 \pm 0.0040$ \\
\hline 0.55 & 926 & 1435 & 2224 & 1649 & $0.1266 \pm 0.0133$ & $0.0198 \pm 0.0021$ & $0.0038 \pm 0.0004$ & $0.0111 \pm 0.0012$ & $0.0856 \pm 0.0206$ & $0.0230 \pm 0.0055$ & $0.0062 \pm 0.0015$ & $0.0152 \pm 0.0036$ \\
\hline 0.75 & 1060 & 1854 & 3245 & 2248 & $0.2019 \pm 0.0217$ & $0.0174 \pm 0.0019$ & $0.0021 \pm 0.0002$ & $0.0078 \pm 0.0008$ & $0.1102 \pm 0.0326$ & $0.0206 \pm 0.0061$ & $0.0038 \pm 0.0011$ & $0.0115 \pm 0.0034$ \\
\hline 0.9 & 1125 & 2137 & 4061 & 2698 & $0.2949 \pm 0.0325$ & $0.0162 \pm 0.0018$ & $0.0015 \pm 0.0002$ & $0.0061 \pm 0.0007$ & $0.1320 \pm 0.0470$ & $0.0193 \pm 0.0069$ & $0.0028 \pm 0.0010$ & $0.0096 \pm 0.0034$ \\
\hline 1.0 & 1156 & 2313 & 4625 & 2998 & $0.3877 \pm 0.0435$ & $0.0156 \pm 0.0017$ & $0.0012 \pm 0.0001$ & $0.0053 \pm 0.0006$ & $0.1483 \pm 0.0612$ & $0.0185 \pm 0.0077$ & $0.0023 \pm 0.0010$ & $0.0085 \pm 0.0035$ \\
\hline
\end{tabular}




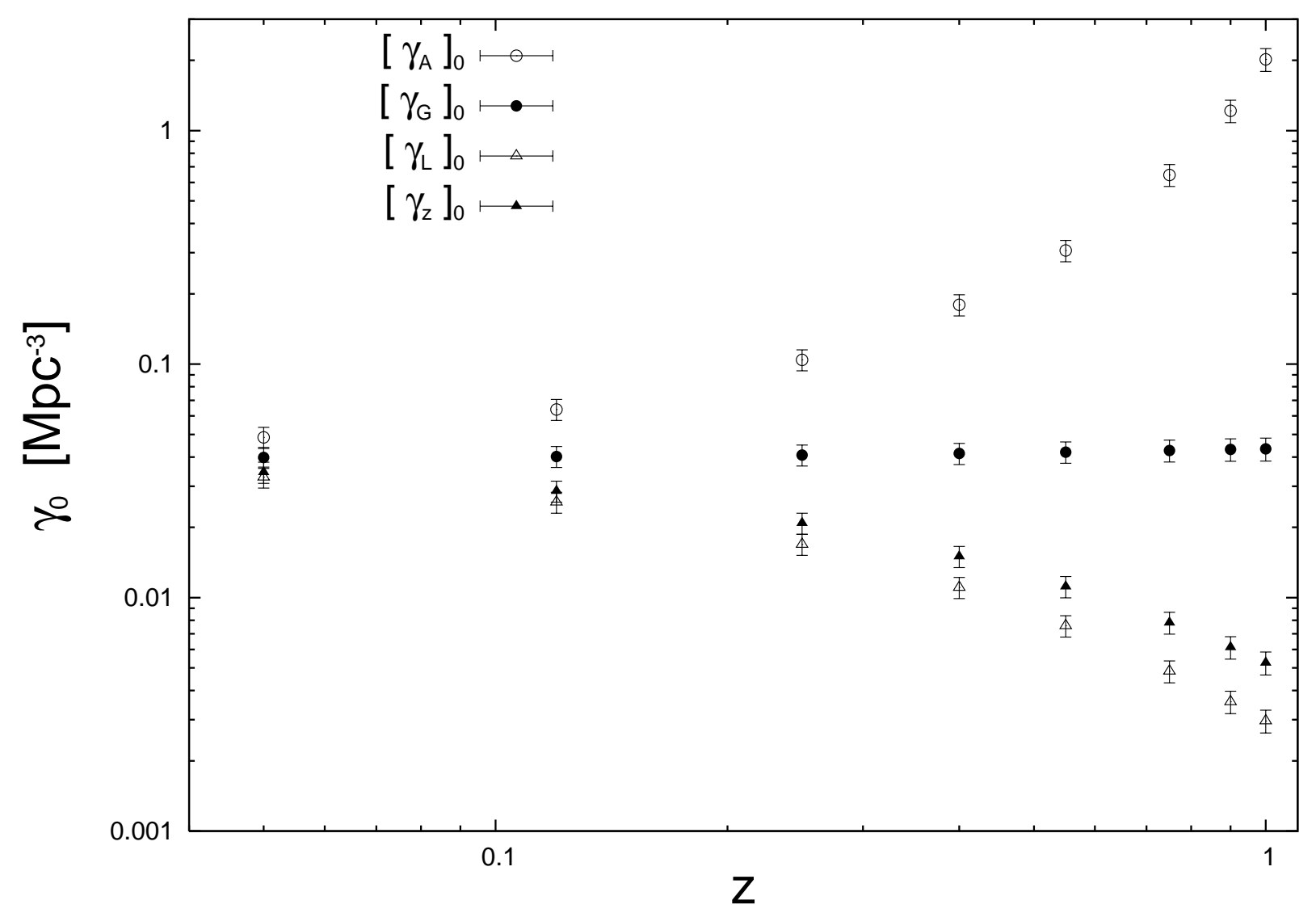

Fig. 1.- Differential densities $\gamma_{0}$ against redshift in the EdS model with CNOC2 data. The same theoretical behavior of the differential count presented in Ribeiro (2005) is preserved with the CNOC2 differential number counting data. Notice the power law pattern when $z>0.1$ for densities obtained with $d_{L}$ and $d_{z}$. 


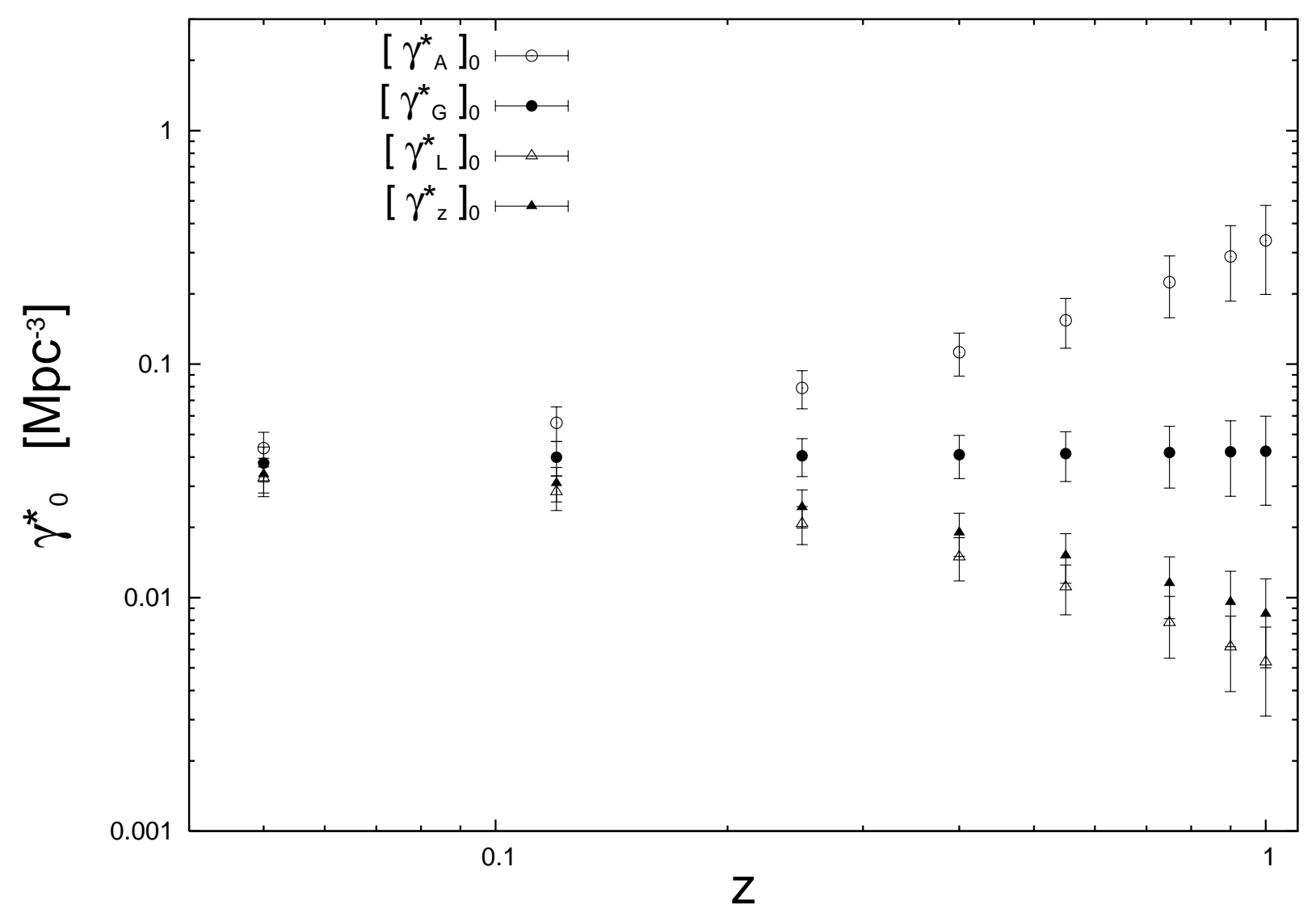

Fig. 2.- Integral differential densities $\gamma_{0}^{*}$ against redshift in the EdS model. As in the previous plot, the critical dependence on the choice of distance is preserved in the CNOC2 data, as well as the visible power law decaying pattern occurring when one chooses $d_{L}$ and $d_{z}$ as distances. 


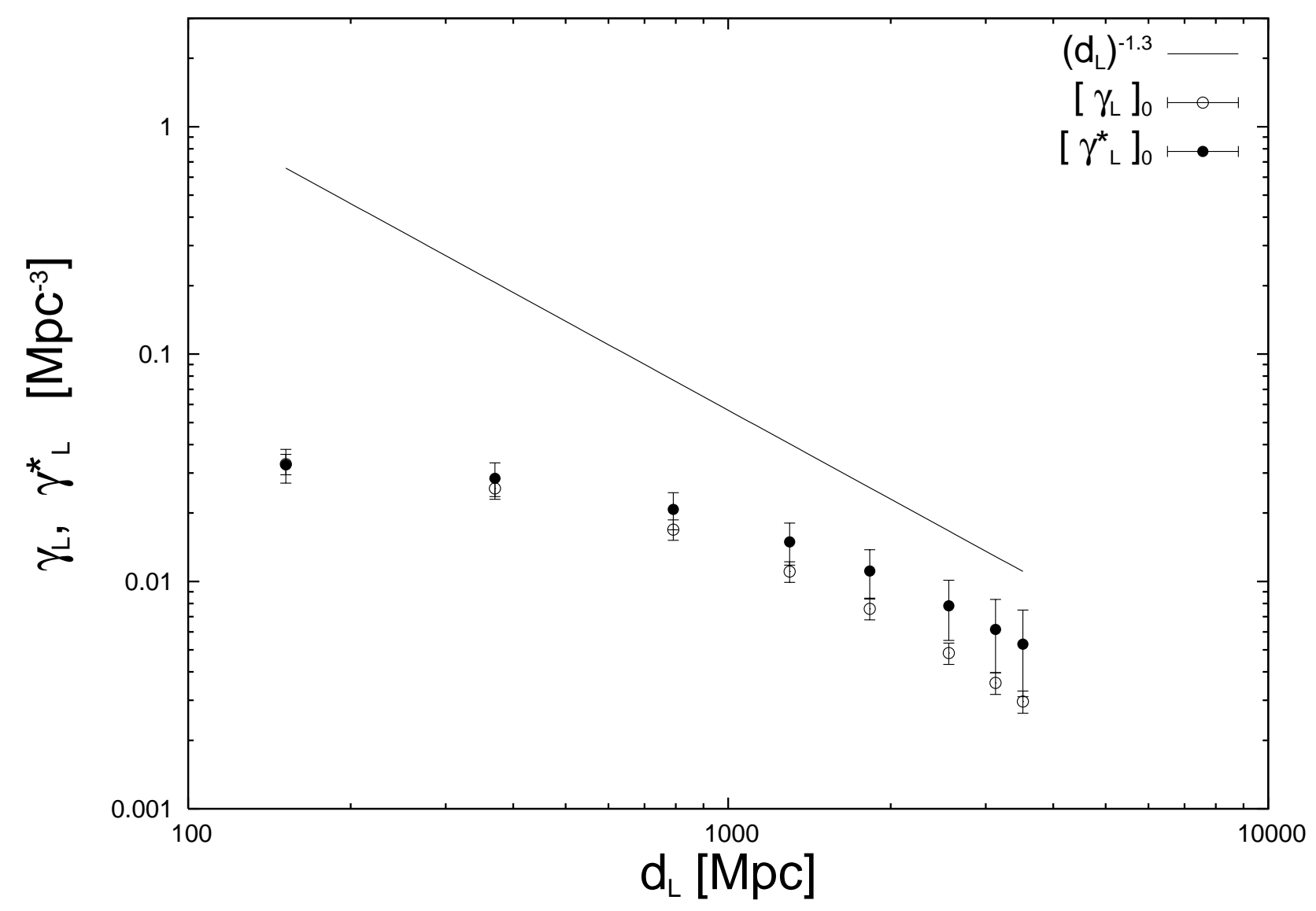

Fig. 3.- Plot of the differential densities obtained from the CNOC2 survey against the luminosity distance in EdS cosmology. Both densities decrease at higher $d_{L}$ and show a clear power law behavior in the tail. The line is for reference only, but its slope is such that in the language of fractals it would mean a fractal dimension $D=1.7$. This plot does not prove that the galaxy distribution as presented in the CNOC2 survey data follows a fractal pattern, but that such an observationally self-similar structure is possible at least in some scales. 


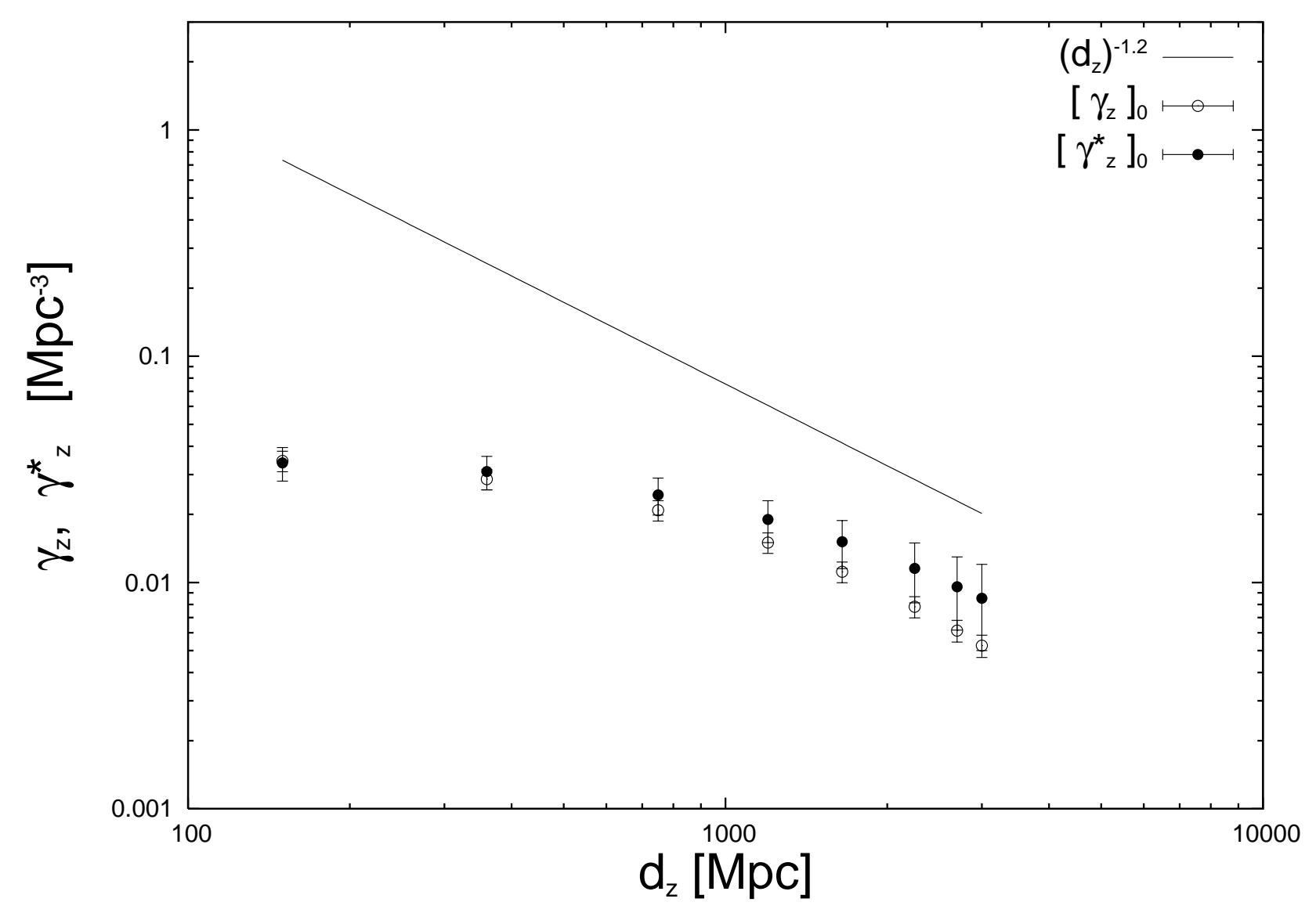

Fig. 4.- Plot of the differential densities obtained from the CNOC2 survey against the redshift distance in EdS cosmology. Similarly to the previous graph, both densities tend to follow a power law decay at higher distances. The straight line is for reference only, but its slope is such that it would mean $D=1.8$. Again, one must remain cautious regarding interpreting this self-similar behavior in the tail as evidence of a fractal pattern. However, the plot indicates that this possibility should not be ruled out either. Further investigation with other samples is clearly required to clarify this point. 


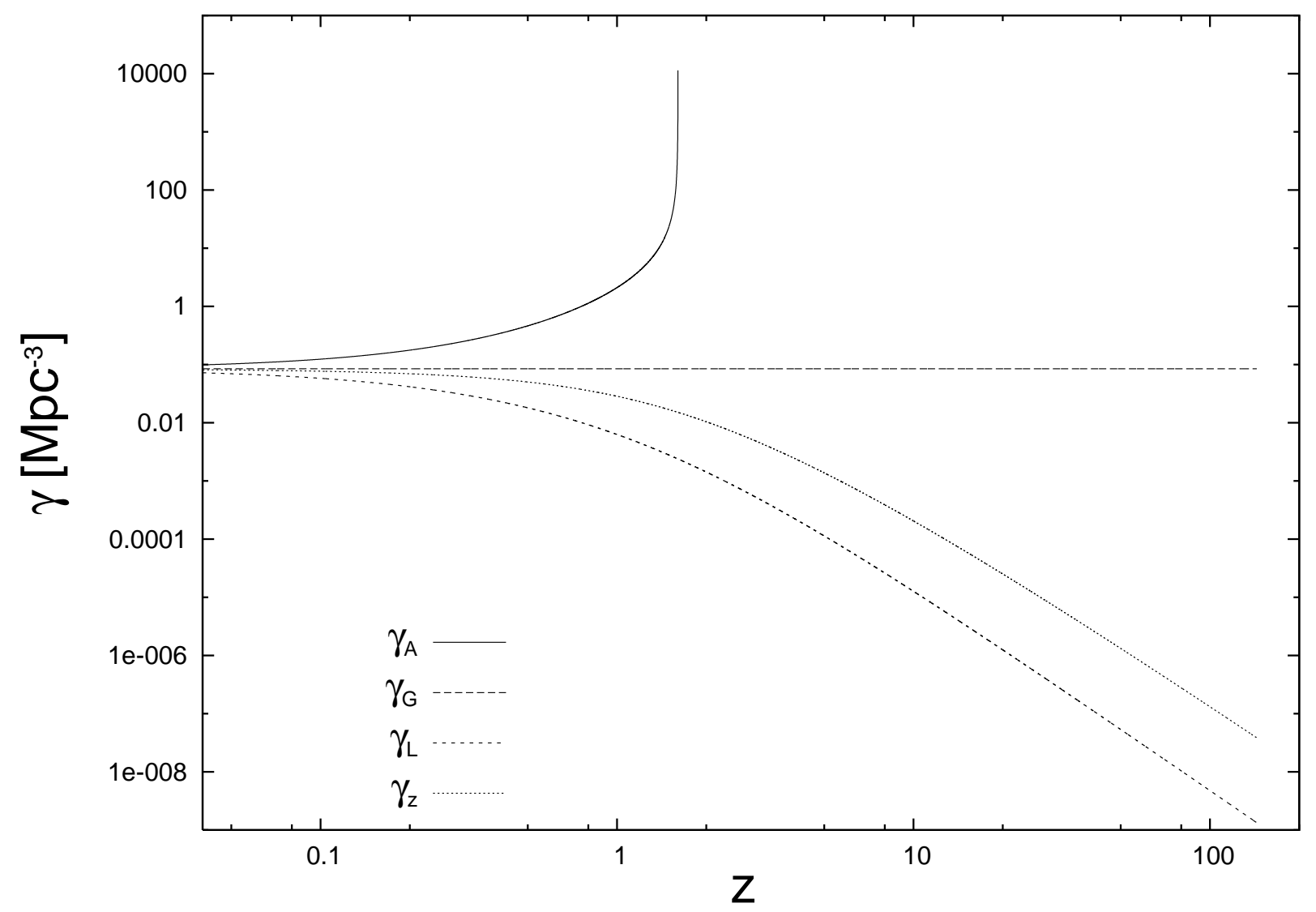

Fig. 5.- Plot of the theoretical differential density $\gamma$ for each observational distance in the $\Omega_{m_{0}}=0.3, \Omega_{\Lambda_{0}}=0.7$ standard cosmology. The results are similar to those of EdS model as shown in Ribeiro (2005) and are presented here for comparison with the observationally derived $\gamma_{0}$ shown in figure 7 . Notice that similarly to the EdS case $\gamma_{A}$ blows up at about $z \approx 1.5$, but that in itself does not mean that the volume defined with $d_{A}$ is incorrect, but only that the way $\gamma_{A}$ is defined has a singularity, which has no special physical significance other than that the distance it involves has a critical point. 


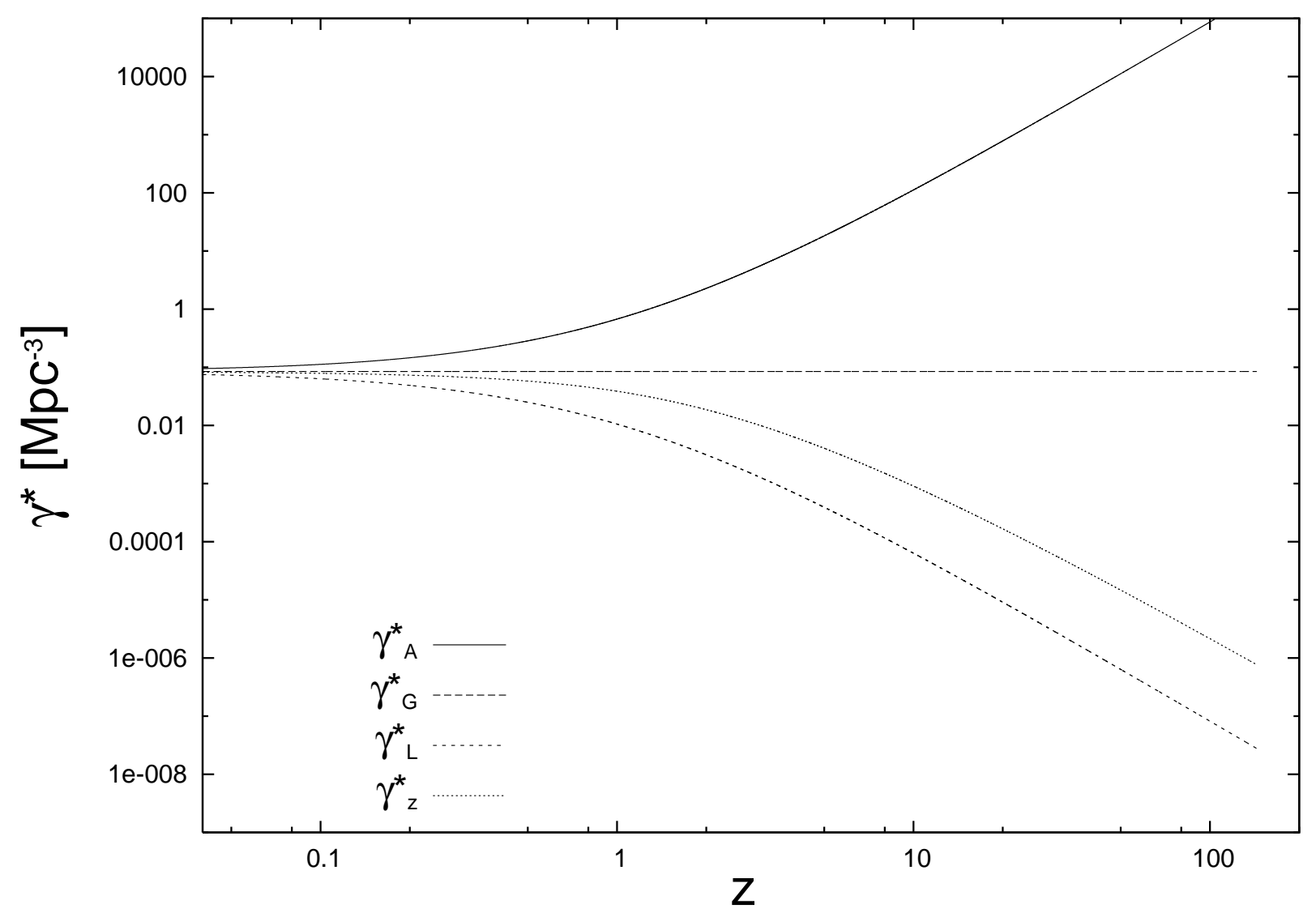

Fig. 6.- Plot of the theoretical integral differential density $\gamma^{*}$ for each observational distance in the $\Omega_{m_{0}}=0.3, \Omega_{\Lambda_{0}}=0.7$ standard cosmological model. The results are similar to those of EdS cosmology (Ribeiro 2005). They are presented here so that one can compare with the observationally derived $\gamma_{0}^{*}$ plotted in figure 8 . 


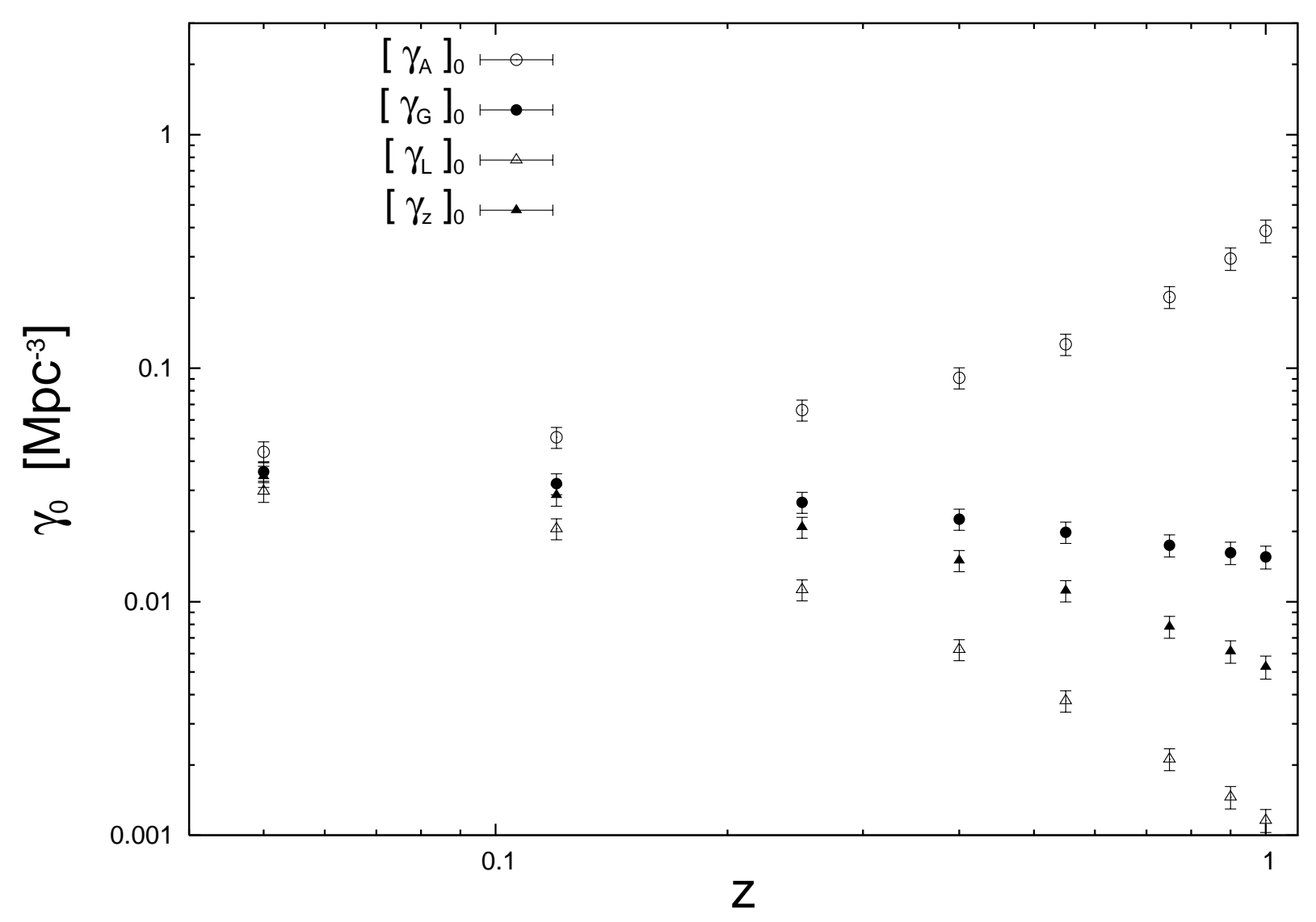

Fig. 7.- This figure shows the observed differential density $\gamma_{0}$ vs. the redshift in the $\Omega_{m_{0}}=0.3, \Omega_{\Lambda_{0}}=0.7$ standard cosmology with CNOC2 differential number counts data of table 4. Notice that similarly to the results shown in figure 1 there is as power law pattern at the tail of the plot for both $d_{L}$ and $d_{z}$. However, the differential density calculated with the galaxy area distance $d_{G}$ does not remain unchanged, but also decays at higher $z$, an effect that can only be explained because this plot uses the CNOC2 differential number count $[d N / d z]_{0}$ for calculating $\gamma_{0}$, rather than its theoretically derived values used in figure 5 . Therefore, it is possible that either the CNOC2 survey is undercounting galaxies at larger $z$ or that its LF source evolution equation is not correct. A third, less likely, possibility is that there could be real spatial inhomogeneity and in this case the FLRW model would not be correct. 


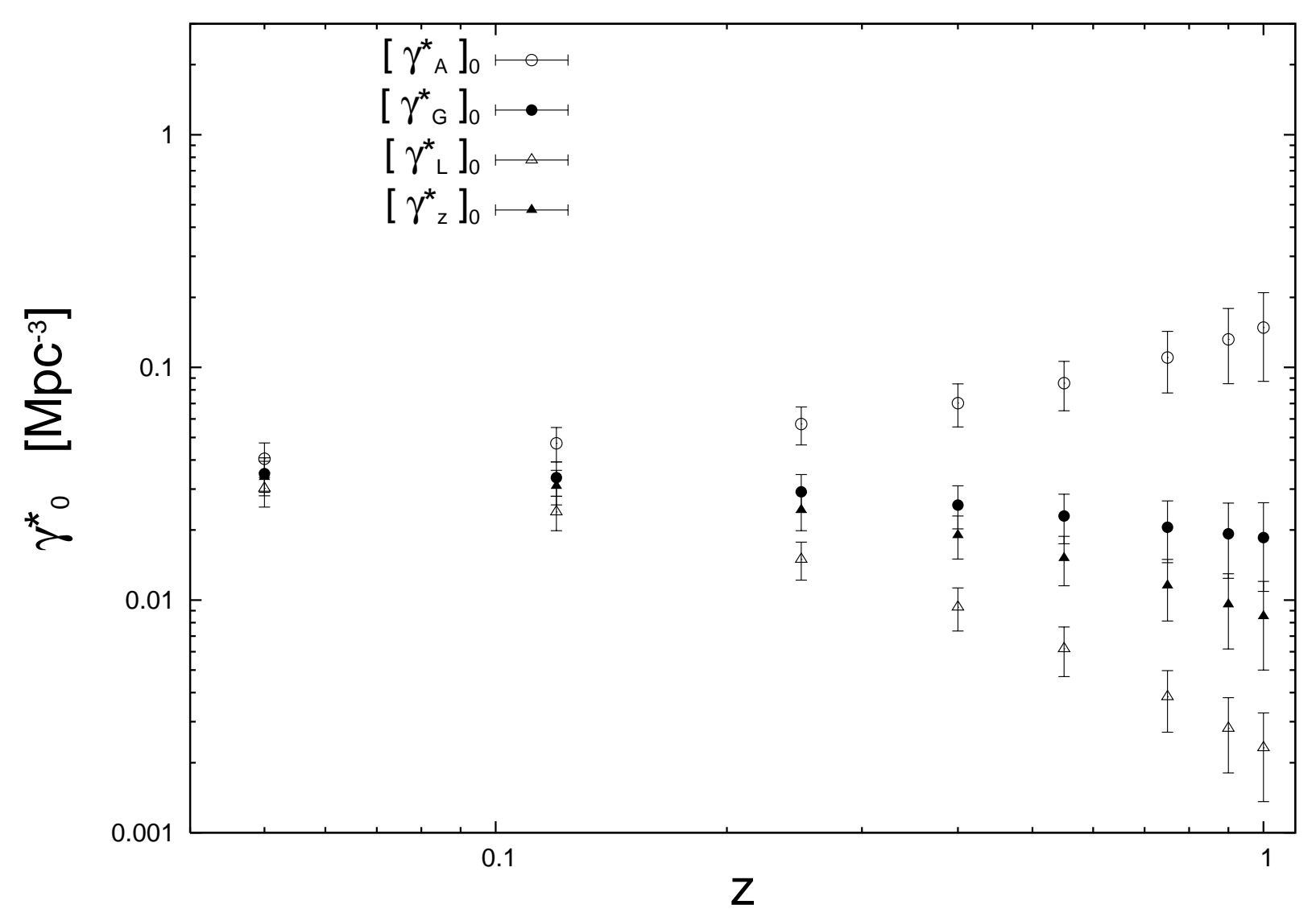

Fig. 8.- Plot of the observed integral differential density $\gamma_{0}^{*}$ against the redshift in the $\Omega_{m_{0}}=0.3, \Omega_{\Lambda_{0}}=0.7$ standard cosmology with a CNOC2 LF derived $[d N / d z]_{0}$. As in the previous plot one can also notice a power law pattern for both the luminosity distance $d_{L}$ and redshift distance $d_{z}$. But, still as in the previous plot, $\gamma_{0}^{*}$ calculated with $d_{G}$ decreases at higher $z$, departing from the theoretical behavior presented in figure 6 . 


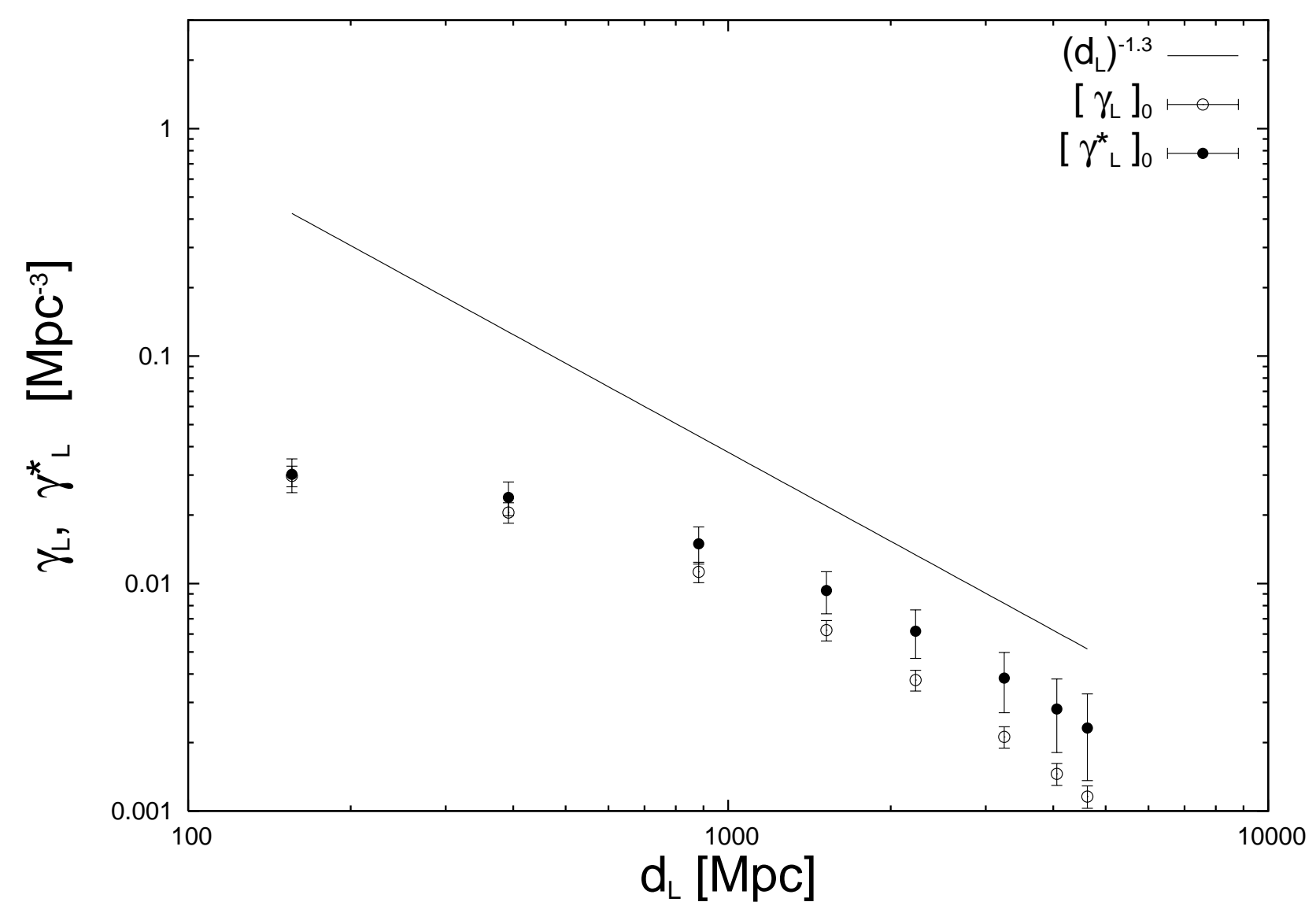

Fig. 9.- Differential densities against the luminosity distance in the $\Omega_{m_{0}}=0.3, \Omega_{\Lambda_{0}}=0.7$ standard cosmology using the CNOC2 differential number count data of table 4 . The power law behavior is visible in the $z>0.1$ ranges. The straight line is for reference only, but its slope would mean a fractal dimension of approximately $D=1.7$. As cautioned above, one must remain careful in interpreting this pattern as evidence of a fractal structure, since the results were obtained only with the CNOC2 survey data. 


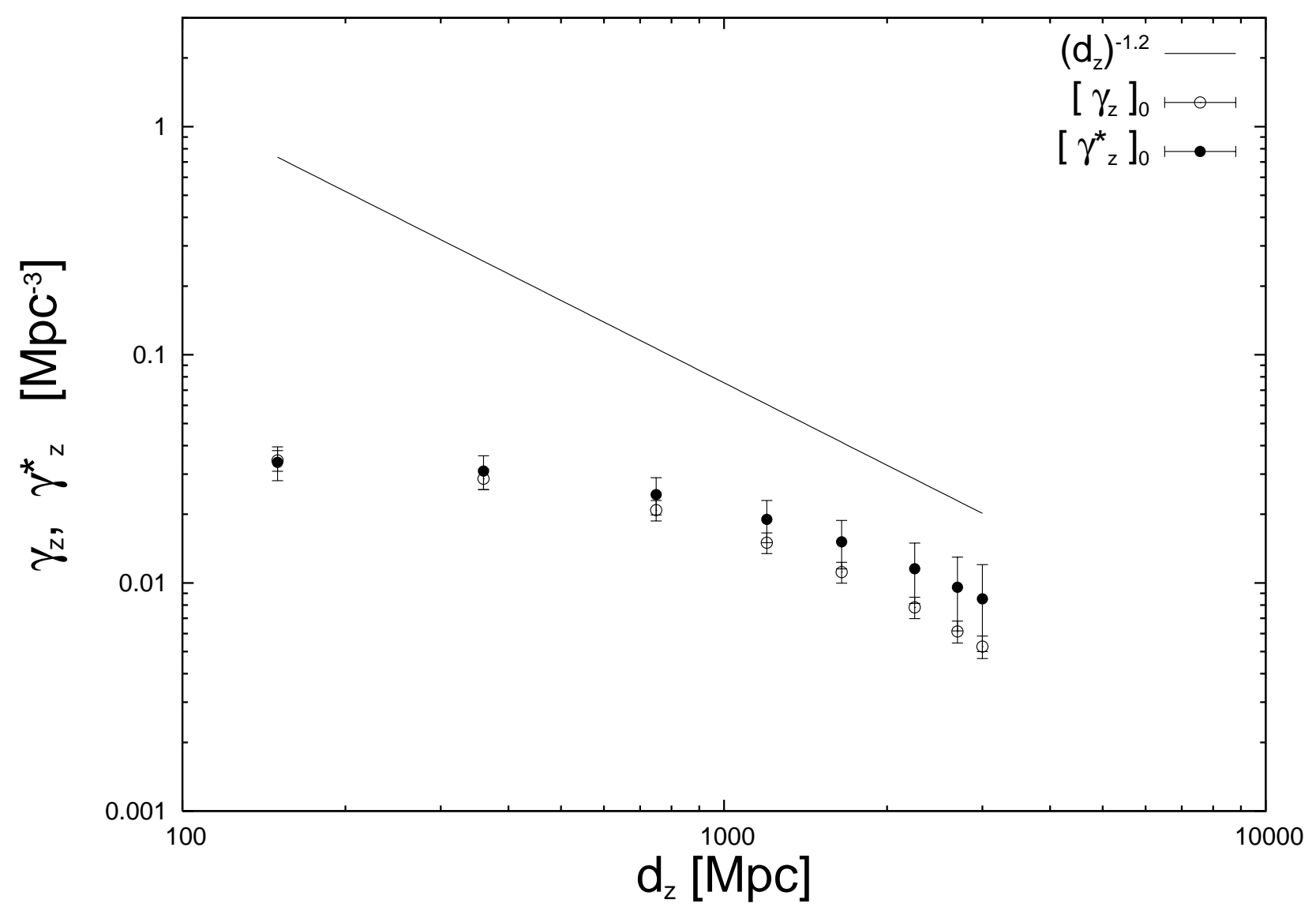

Fig. 10.- Differential densities versus the redshift in the $\Omega_{m_{0}}=0.3, \Omega_{\Lambda_{0}}=0.7$ standard cosmological model where $[d N / d z]_{0}$ was derived from the LF obtained with the CNOC2 galaxy survey (see table 4). Similarly to the previous figure, one can notice a power law pattern in the tail of both plots. The straight line is for reference only, but would mean approximately $D=1.8$ if one interprets these results as possible evidence of a fractal structure for $z>0.1$ ranges. 